\title{
Burundi: Second Review Under the Three-Year Arrangement Under the Poverty Reduction and Growth Facility and Request for Modification of Performance Criteria-Staff Report; Press Release on the Executive Board Discussion; and Statement by the Executive Director for Burundi
}

In the context of the second review under the three-year arrangement under the Poverty Reduction and Growth Facility and a request for modification of performance criteria, the following documents have been released and are included in this package:

- $\quad$ The staff report for Second Review Under the Three-Year Arrangement Under the Poverty Reduction and Growth Facility and Request for Modification of Performance Criteria, prepared by a staff team of the IMF, following discussions that ended on June 10, 2009, with the officials of Burundi on economic developments and policies. Based on information available at the time of these discussions, the staff report was completed on June 29, 2009. The views expressed in the staff report are those of the staff team and do not necessarily reflect the views of the Executive Board of the IMF.

- $\quad$ A Press Release summarizing the views of the Executive Board as expressed during its July 13, 2009 discussion of the staff report that completed the review.

- $\quad$ A statement by the Executive Director for Burundi.

The documents listed below have been or will be separately released.

Letter of Intent sent to the IMF by the authorities of Burundi* Memorandum of Economic and Financial Policies by the authorities of Burundi* Technical Memorandum of Understanding*

*Also included in Staff Report

The policy of publication of staff reports and other documents allows for the deletion of market-sensitive information.

\author{
Copies of this report are available to the public from \\ International Monetary Fund • Publication Services \\ $70019^{\text {th }}$ Street, N.W. • Washington, D.C. 20431 \\ Telephone: (202) 623-7430 • Telefax: (202) 623-7201 \\ E-mail: publications@imf.org •Internet: http://www.imf.org
}

\section{International Monetary Fund Washington, D.C.}



INTERNATIONAL MONETARY FUND

BURUNDI

\title{
Second Review Under the Three-Year Arrangment Under the Poverty Reduction and Growth Facility and Request for Modification of Performance Criteria
}

\author{
Prepared by the African Department \\ (In consultation with other departments)
}

Approved by Robert Sharer and Dominique Desruelle

June 29, 2009

\section{PRGF arrangement:}

Discussions:

Program review:

Publication:
On July 7, 2008, the Executive Board approved a three-year PRGF arrangement with access of SDR 46.2 million ( 60 percent of quota). The first review of the PRGF-supported program was completed on January 28, 2009, along with the HIPC completion point decision.

A mission comprising Messrs. Akitoby (head), Ioannou, Thomas, and Camara (all AFR) visited Bujumbura May 27-June 10, 2009, to conduct the second review of the PRGF-supported program. The mission coordinated with World Bank and African Development Bank missions.

The staff met with President Nkurunziza, First Vice-President Sahinguvu, Second Vice-President Ntisezerana, Minister of Finance Nizigama, Central Bank Governor Sindayigaya, other senior government officials, and representatives of labor unions, the private sector, NGOs, the donor community, and the media.

All quantitative and structural performance criteria for March 2009 were met, and structural reforms are on track. Staff recommends completion of the second review.

The authorities consent to the publication of this report, the letter of intent, and the MEFP. 


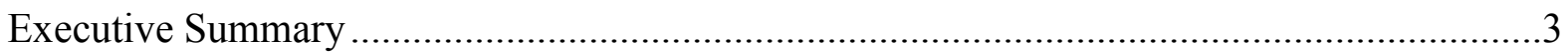

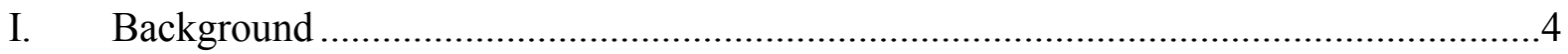

II. Recent Developments and Performance Under the PRGF .........................................4

III. Consolidating Economic Stability and Sustaining Growth........................................6

A. Macroeconomic Outlook .............................................................................6

B. Fiscal Policy and Related Reforms ............................................................... 7

C. Monetary Policy ........................................................................................ 9

D. External Sector Policies ................................................................................ 9

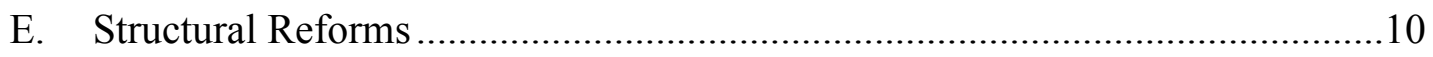

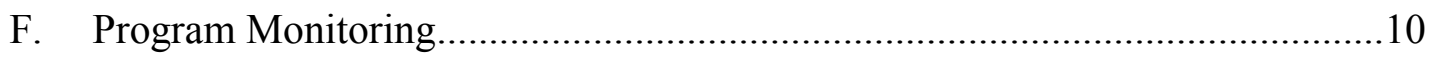

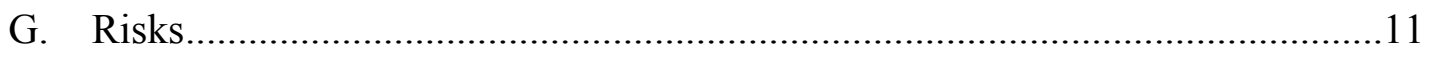

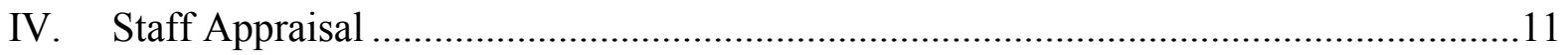

Figure

1. Recent Macroeconomic Performance, 2000-08 …........................................................5

Text Table

1. Medium Term Outlook 2008-12 …........................................................................ 7

Tables

1. Millennium Development Goals ............................................................................. 13

2. Selected Economic and Financial Indicators, 2007-12 ….....................................14

3. General Government Operations, 2007-12 …......................................................... 15

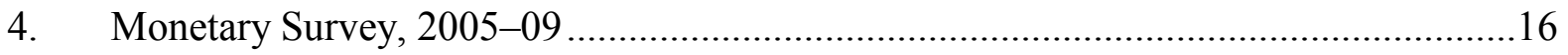

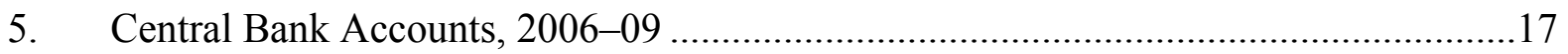

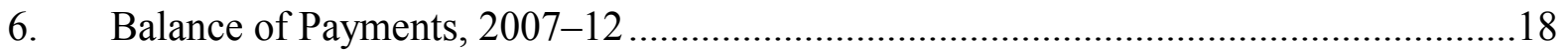

7. Banking System Soundness Indicators, 2005-09 .....................................................19

8. Actual and Projected Schedule of PRGF Disbursements and Reviews, 2008-11 _.......20

Appendix

1. Letter of Intent................................................................................................2 21

Attachment I. Memorandum of Economic and Financial Policies ....................23

Attachment II. Technical Memorandum of Understanding ................................32

Appendix Tables

I.1. Performance Criteria and Indicative Targets for 2008 ........................................29

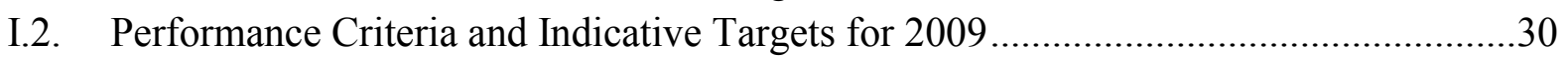

I.3. Performance Criteria and Structural Benchmarks for 2009 ......................................31 


\section{EXECUTIVE SUMMARY}

Burundi's economy performed well during 2008, though food and fuel price shocks kept inflation high. Real GDP growth increased to $4 \frac{1}{2}$ percent, mainly because of a good coffee harvest and more donor-financed projects. With international commodity prices higher in the first half of the year, 12-month inflation peaked at about 30 percent in June before declining to about 26 percent by year-end. Higher donor support helped build gross official reserves to about $6 \frac{1}{2}$ months of imports. The real effective exchange rate appreciated by 16 percent.

In a difficult post-conflict environment, performance under the PRGF-supported program was generally satisfactory. Most indicative targets for end-December 2008 were met, as were all quantitative and structural performance criteria for March 2009, and structural reforms are on track. In particular, the value-added tax law was promulgated, payroll management was transferred to the Ministry of Finance, and the census of government employees was completed. Monetary and financial sector reforms have also progressed well, and the foreign exchange auction system is being revamped.

Though Burundi's macroeconomic prospects for 2009 remain positive, they are less so than they were because of the global financial crisis. Projected economic growth would moderate to 3.2 percent, from $4 \frac{1}{2} 2$ percent in 2008 , mainly because of (i) reduced demand for exports; (ii) a decline in world coffee prices; and (iii) lower private transfers and foreign direct investment. Against the backdrop of weak economic growth and lower international oil and food prices, 12-month inflation is expected to decline to 9 percent by year-end.

The discussions focused on ways to mitigate the adverse impact of the global economic slowdown on Burundi. Fiscal policy will be eased by about 1.3 percent of GDP to accommodate the temporary revenue shortfall due to the global financial crisis. The revised fiscal program also incorporates additional grants-financed spending of 1.5 percent of GDP to mitigate the impact of the crisis on the poor. Monetary policy will be eased slightly, while still seeking to reduce inflation to single digits. The flexible exchange rate regime will also help the economy adjust.

Because Burundi is a postconflict country, risks to the program remain significant. Foremost would be a worsening political, social, and security situation as general elections near in mid-2010. Other risks are governance slippages and worse than expected effects of the global financial crisis.

Staff recommends completion of the second review, based on Burundi's performance and the strength of the program. 


\section{BACKGROUND}

1. Burundi is one of the least developed countries in the world. GDP per capita is about US\$140, and about 67 percent of the population lives below the poverty line. While the country is making some progress toward the Millennium Development Goals (MDGs), it is unlikely that any will be achieved by 2015 (Table 1).

2. The country is emerging from more than a decade of civil conflict. In December 2008, a power-sharing agreement was signed with the last rebel group, Forces Nationales pour la Libération (FNL). FNL is now recognized as a political party, and the rebels are being reintegrated into national institutions. However, security remains fragile ahead of the general elections scheduled for mid- 2010 .

3. The World Bank in August 2008 approved a new country assistance strategy that focuses on structural reforms to further increase growth and reduce poverty. The main priorities are to: (i) promote sustainable and broad-based economic growth; and (ii) improve access to social services and consolidate social stability. The World Bank-IMF Work Program is described in the Informational Annex.

\section{Recent Developments And Performance Under the PRGF}

4. Economic growth accelerated and inflation increased in 2008 (Figure 1 and Table 2). Real GDP growth increased to $4 \frac{1}{2}$ percent, up from 3.6 percent in 2007, mainly because of a good coffee harvest and more donor-financed investment projects. With international commodity prices higher in the first half of the year, 12-month inflation peaked at about 30 percent in June 2008 before declining to 26 percent by year-end, far above the 14 percent target. In 2008 the nominal exchange rate depreciated by 10 percent, but the real effective exchange rate appreciated by 16 percent. $^{1}$

5. Performance under the PRGF-supported program was generally satisfactory in 2008 (MEFP, Table I.1). The central bank met the end-year indicative targets on net foreign assets and net domestic assets comfortably The indicative target on net domestic financing of the government was missed by about 0.4 percent of GDP because of higher domestically financed spending than was programmed.

\section{All quantitative and structural performance criteria for March 2009 were} observed, and structural reforms are on track. With regard to fiscal reforms, the valueadded tax (VAT) law was promulgated, payroll management was transferred to the Ministry of Finance (performance criterion), the census of government employees was completed, and adoption of the PFM strategy laid the groundwork for PFM reform. Monetary and financial sector reforms have also progressed well (MEFP, ๆ9-10).

\footnotetext{
${ }^{1}$ The appreciation of the real effective exchange rate reflects the impact of higher food and oil prices on inflation. Discussions with the authorities and the private sector suggest that the appreciation has only a limited impact on competitiveness.
} 
Figure 1. Recent Macroeconomic Performance, 2000-08

Growth was volatile but trended upward...

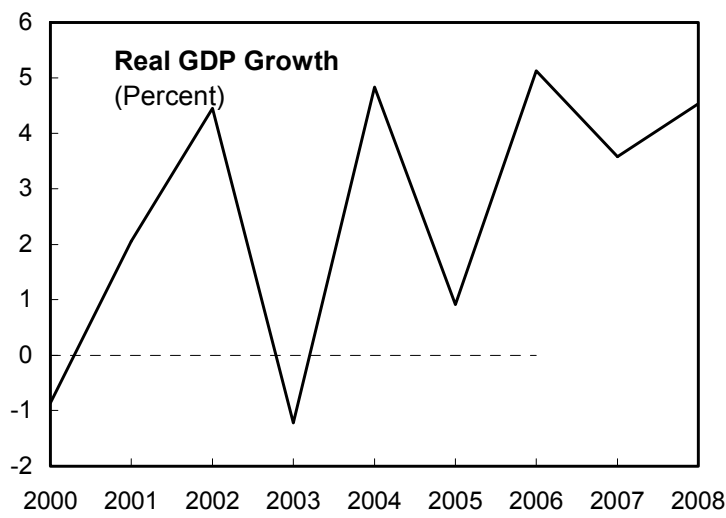

The fiscal position has improved...

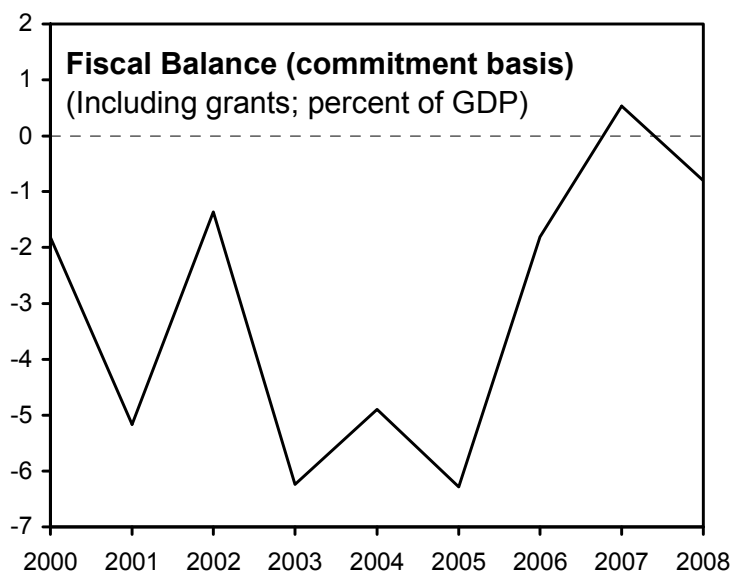

The current account deteriorated reflecting higher donor-financed imports...

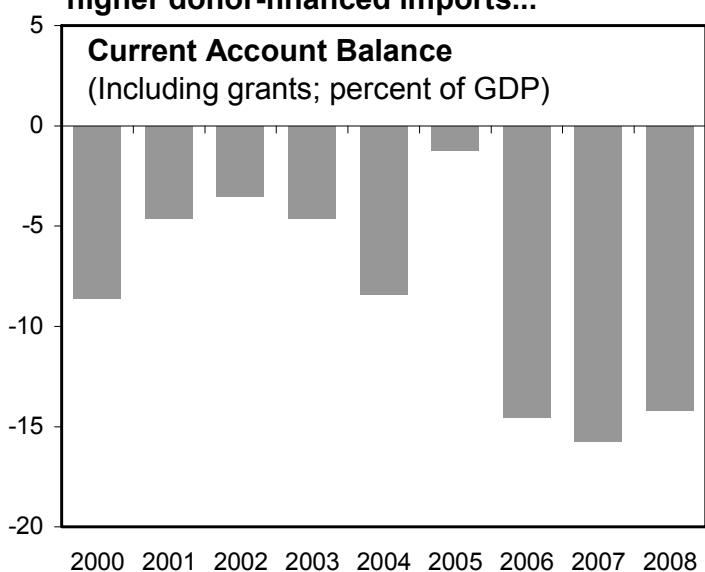

Sources: Burundi authorities; and IMF staff estimates. ...while inflation has accelerated sharply.

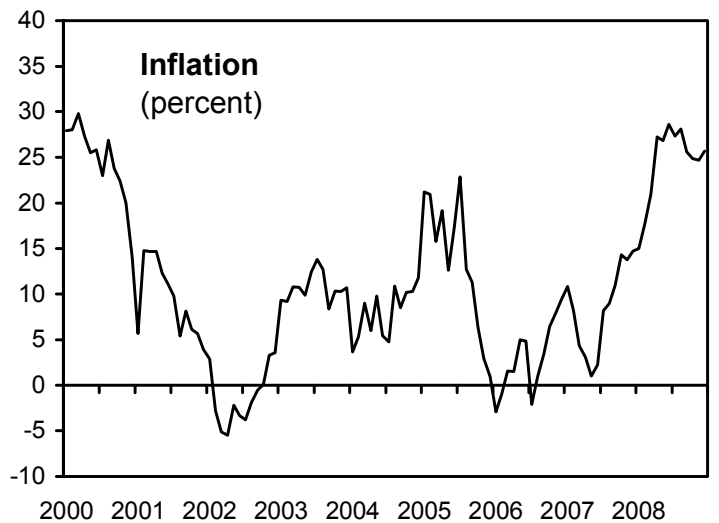

... as external grants have increased.

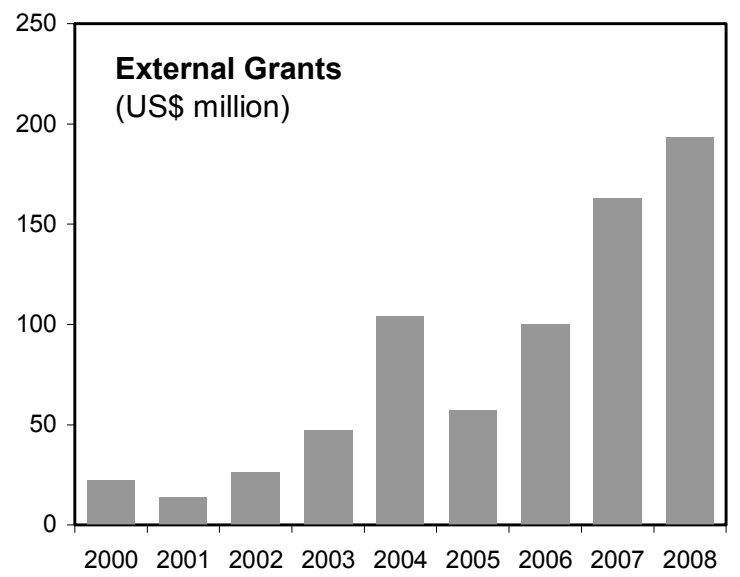

...but official inflows helped rebuild international reserves.

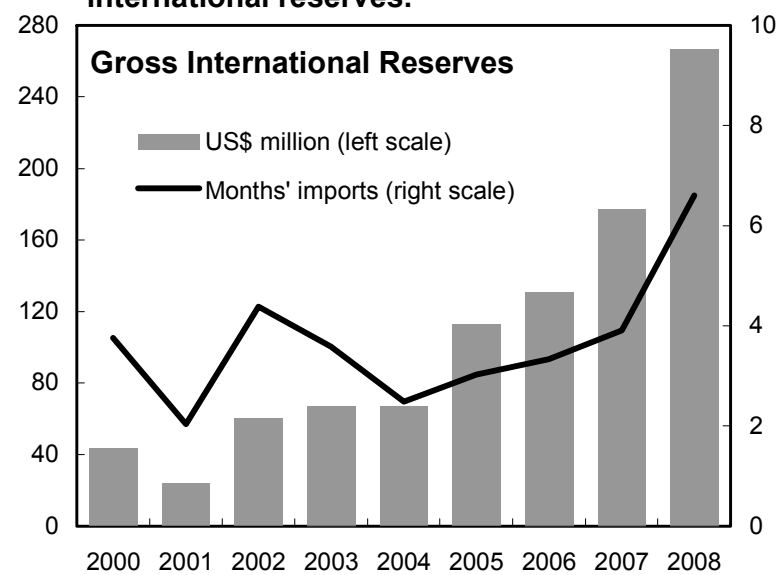




\section{Consolidating Economic StabiLITY AND Sustaining Growth}

7. The discussions focused on appropriate policy responses to the global financial crisis, with a view to consolidating economic stability and further reducing poverty. Fiscal and monetary policies will be prudent, and the exchange rate regime will remain flexible. In particular, fiscal policy will be eased to accommodate the temporary revenue shortfall caused by the global crisis. Given the confluence of a more favorable inflation outlook and weaker growth for 2009, monetary policy will also be eased slightly to help the economy adjust to the shocks from the global financial crisis while still seeking to reduce inflation to single digits.

\section{A. Macroeconomic Outlook}

8. Though Burundi's macroeconomic prospects for 2009 remain positive, they are less so than they were because of the global financial crisis. Economic growth is projected to moderate to 3.2 percent in 2009 , from $4 \frac{1}{2}$ percent in 2008 , mainly because of (i) reduced demand for exports; (ii) a decline in world coffee prices; and (iii) lower private transfers and foreign direct investment. However, weak economic growth and lower international oil and food prices have improved the inflation outlook for 2009. Between December 31, 2008, and April 30, 2009, 12-month inflation fell from 26 percent to 13 percent; ${ }^{2}$ by year-end it is expected to decline further to 9 percent.

9. Provided the security situation continues to improve, Burundi's medium-term economic outlook appears to be positive (Text Table 1). GDP growth is expected to average about 4 percent over 2009-12, driven by three main factors: (1) continued removal of major economic distortions, especially in the coffee sector, which will boost total factor productivity; (2) a substantial increase in aid-financed investment, largely for infrastructure renovation, which will help relieve major supply bottlenecks; and (3) the impact of accession to the East African Community (EAC), which will help diversify the economy, stimulate competition, and attract more investment.

\footnotetext{
${ }^{2}$ Over the same period, 12-month food price inflation declined from 40 percent to 17 percent. Food prices account for about 52 percent of the overall price index.
} 
Text Table 1. Burundi: Medium-Term Outlook, 2008-12

\begin{tabular}{|c|c|c|c|c|c|c|c|}
\hline & \multicolumn{2}{|c|}{2008} & \multicolumn{2}{|c|}{2009} & \multirow{2}{*}{2010} & \multirow{2}{*}{$\begin{array}{l}2011 \\
\text { Proj. }\end{array}$} & \multirow[t]{2}{*}{2012} \\
\hline & Prog. & Est. & Prog. & $\begin{array}{r}\text { Rev. } \\
\text { Prog. }\end{array}$ & & & \\
\hline & \multicolumn{7}{|c|}{ (Annual percentage change, unless otherwise indicated) } \\
\hline \multicolumn{8}{|l|}{ National income and prices } \\
\hline Real GDP growth & 4.5 & 4.5 & 4.5 & 3.2 & 3.6 & 4.2 & 4.8 \\
\hline Consumer prices (end of period) & 14.0 & 25.7 & 9.0 & 9.1 & 7.5 & 6.0 & 5.5 \\
\hline & \multicolumn{7}{|c|}{ (Percent of GDP, unless otherwise indicated) } \\
\hline \multicolumn{8}{|l|}{ General government } \\
\hline Revenue (excluding grants) & 19.1 & 18.6 & 19.3 & 18.0 & 18.6 & 19.3 & 19.5 \\
\hline Total expenditure and net lending & 42.6 & 44.1 & 44.6 & 46.1 & 45.5 & 41.8 & 41.2 \\
\hline \multicolumn{8}{|l|}{ Overall balance } \\
\hline Commitment basis (after non-HIPC grants) & -3.6 & -3.8 & -3.0 & -4.1 & -4.0 & -1.9 & -1.3 \\
\hline Cash basis (after non-HIPC grants) & -4.9 & -4.6 & -3.0 & -4.3 & -4.0 & -1.9 & -1.3 \\
\hline \multicolumn{8}{|l|}{ External sector } \\
\hline Current account balance & -18.2 & -14.2 & -11.5 & -9.5 & -10.6 & -13.6 & -14.7 \\
\hline Overall balance of payments & -1.1 & 7.8 & -1.0 & -2.5 & 0.1 & -0.9 & -0.8 \\
\hline \multicolumn{8}{|l|}{ Gross official reserves } \\
\hline In US\$ millions & 155.3 & 266.6 & 153.8 & 231.8 & 247.7 & 249.8 & 252.1 \\
\hline In months of imports & 3.2 & 6.6 & 3.4 & 5.5 & 5.0 & 4.5 & 4.0 \\
\hline
\end{tabular}

Sources: Burundi authorities; and IMF staff estimates and projections.

\section{B. Fiscal Policy and Related Reforms}

10. The revised program targets an overall fiscal deficit (on a cash basis, and including non-HIPC grants) of 4.3 percent of GDP, compared with the 3 percent envisaged previously, because of the temporary revenue loss caused by the global financial crisis. Total spending would increase by $1 \frac{1}{2}$ percent of GDP, reflecting grants-financed outlays to mitigate the impact of the crisis on the poor. The mission supported the authorities' decision to finance the temporary increase in the fiscal deficit by (i) delaying repayment of past central bank advances ( 0.6 percent of GDP) and (ii) accessing limited and temporary central bank financing ( 0.7 percent of GDP). The envisaged increase in central bank financing is consistent with both macroeconomic stability and the new central bank charter. ${ }^{3}$

11. Fiscal policy is also geared to addressing debt sustainability concerns. Given Burundi's debt burden, external financing of the budget should be strictly limited to grants and highly concessional loans. Planned fiscal reforms over 2009-11 will also help ensure debt sustainability. These include the new budget law and the reforms of tax policy, revenue administration, expenditure policy, treasury management, and debt management (IMF Country Report No. 08/282 and IMF Country Report No. 09/93).

\footnotetext{
${ }^{3}$ The additional central bank financing poses minimal risk to the inflation target given the economic slowdown and low international oil and food prices.
} 
12. Fiscal policy will continue to support the use of aid. If concessional external financing is higher than expected, the program will accommodate a higher fiscal deficit by allowing increased spending in areas critical to meeting the MDGs. The authorities are continuing to work with the World Bank to improve Burundi's absorptive capacity.

\section{The authorities recognize that mobilizing domestic revenue is critical for} medium-term fiscal sustainability and increased poverty-reducing expenditure. The revised revenue target for 2009 is 18 percent of GDP, still above the average of 17.3 percent of GDP for fragile states. In view of the negative impact of the global financial crisis on revenue, staff urged the authorities to continue their efforts to broaden the revenue base by reducing exemptions and strengthening tax and customs administration. Moreover, with Burundi's accession to the EAC, the authorities will introduce in July 2009 a VAT to replace a transaction tax and the EAC common external tariff.

\section{The authorities are committed to reallocating spending to MDG-related sectors,} supported by debt relief from the HIPC Initiative and the Multilateral Debt Relief Initiative (MDRI) (MEFP, $₫ 14,16)$. Spending on priority sectors is expected to increase from 8.8 percent of GDP in 2008 to 9.9 percent of GDP in 2009. In line with Burundi's poverty reduction strategy, MDRI resources will be spent on agriculture, water, rural infrastructure, health, and education.

\section{The revised program takes into account a higher payroll in the health sector.}

Because of new allowances granted to frontline health workers, the wage bill will be revised upward by about 0.5 percent of GDP, which will be offset by cuts in nonpriority spending. The government stressed that the new allowances were needed to stem a persistent outflow of doctors and nurses from the public health system and preserve recent gains in health indicators.

\section{Staff urged the authorities to continue to reform wages and employment. The} authorities reiterated their commitment to pursue planned civil service reforms (MEFP, $\llbracket 16$ ), in consultation with the World Bank. Now that payroll management has been transferred to the Ministry of Finance, the government will audit the payroll to ensure that wage and allowance payments comply with the law. The audit will also provide a sound basis for the reform of wage policy the government is undertaking in consultation with the World Bank.

17. The authorities are committed to reforming PFM (MEFP, $\mid 22$ ). The council of ministers adopted a PFM strategy and action plan, and the government has stepped up rationalization of its bank accounts. This year it has also begun phasing in the new budget organic law. 


\section{Monetary Policy}

18. Monetary policy will continue to be geared to stabilizing prices while allowing sufficient scope for economic growth. It will also be eased slightly to help the economy adjust to the crisis, though without jeopardizing the inflation objective. To secure the singledigit inflation objective, broad money will continue to rise more slowly than nominal GDP. Staff expects that in 2009 reserve money will grow by about $13 \frac{1}{2}$ percent and broad money by about $14 \frac{1}{2}$ percent. Growth in credit to the private sector is expected to moderate because of the economic slowdown.

19. The Ministry of Finance and the central bank agreed that close coordination of fiscal and monetary policies is essential to achieving the inflation target (MEFP, $\mid 19$ ). They recognize that cooperation is needed to improve central bank liquidity forecasts and ensure that aid-financed expenditures are absorbed through sales of foreign exchange.

20. Consequent to the global financial crisis, the central bank is enhancing banking supervision (MEFP, -23). Although the recent Bank-Fund FSAP mission detected no obvious sign of imminent systemic stress, staff urged the authorities to remain vigilant by moving quickly to implement MCM recommendations on banking supervision, particularly those related to risk-based supervision.

\section{External Sector Policies}

\section{External developments in $\mathbf{2 0 0 9}$ are expected to be dominated by the global}

financial crisis. Despite a fall in coffee prices and private transfers, the external current account deficit is expected to improve, mainly because of the projected decline in international food and oil prices (food and oil imports comprise one-fourth of total imports). ${ }^{4}$ Donors are expected to meet their commitments for 2009. Because of lower private sector financing and foreign direct investment, it is expected that the capital and financial account will worsen, the overall balance of payments will register a small deficit, and thus gross official reserves will decline slightly, to about $5 \frac{1}{2}$ months of imports.

\section{Staff stressed that improved foreign exchange markets will help the economy} adjust to exogenous shocks (MEFP, $\mid 20)$. Building on recent progress in launching symmetrical foreign exchange auctions, the authorities will reinforce the auction system while preparing the groundwork for an interbank foreign exchange market in the medium term. Staff urged them to also take steps to eliminate the remaining multiple currency practice and accept the obligations of Article VIII, Section 2, 3, and 4. They intend to request Fund TA for this.

\footnotetext{
${ }^{4}$ Import volume growth will moderate slightly, despite lower prices, because of the economic slowdown.
} 
23. The authorities should continue to firm up management of external debt now that Burundi has reached the HIPC completion point and received MDRI relief. According to the latest low-income country debt sustainability analysis (IMF Country Report No. 09/84), Burundi faces a high risk of debt distress - making it even more important to continue seeking only grants and highly concessional loans.

24. The government has established an interministerial committee to coordinate EAC integration and facilitate decision-making. A national strategy and action plan, prepared with the assistance of development partners, will be adopted soon.

\section{E. Structural Reforms}

25. In addition to the structural reforms discussed (above and MEFP $\mathbb{2 0}$, 22-24), the government is making steady progress on reforming the coffee and oil sectors. Since adopting a comprehensive reform plan for the coffee sector, the authorities have established a regulatory authority and published bidding invitations for the sale of coffee washing stations.

26. The authorities are committed to using a price adjustment mechanism for petroleum products $(\mathrm{MEFP}, \mathbb{1 1}$, 26). The government issued a decree in May 2009 setting out the terms for monthly adjustment of retail prices for petroleum products on the basis of World Bank and Fund TA.

\section{F. Program Monitoring}

27. Semiannual quantitative performance criteria focus on net foreign assets and net domestic assets of the central bank and net domestic financing of the government, with adjusters to deal with aid volatility. There are also three continuous performance criteria: zero ceilings for (i) new nonconcessional external debt contracted or guaranteed; (ii) shortterm external debt; and (iii) accumulation of external arrears. Indicative targets have been established for domestic arrears accumulation and reserve money (MEFP, Table I.2). The authorities are requesting (i) modification of quantitative performance criteria for endSeptember to take into account the impact of the global financial crisis; and (ii) the conversion of all structural performance criteria into structural benchmarks, in line with the recent Board decision on conditionality policy. As foreshadowed in IMF Country Report No. 08/282 ( $(51)$, staff proposes to remove the indicative target on the wage bill because the authorities have fulfilled the structural conditionality on the civil service census and payroll management.

28. The proposed structural conditionality (see MEFP, Table I.3) is linked to the first strategic axis of the Poverty Reduction Strategy Paper, strengthening economic governance, especially bringing transparency to financial management. 


\section{G. Risks}

29. Although the authorities have reiterated their firm commitment to the program and to good governance, there are two main risks:

- $\quad$ First, a worsening political and social situation ahead of next year's general elections would be a major setback. Continued implementation of the recent power-sharing agreement between the government and the last rebel group could help contain this risk.

- $\quad$ Second, the global financial crisis on economic growth could have more impact than expected. Should this risk materialize, the authorities have a contingency plan to cut nonpriority spending if tax revenue is lower (MEFP, \17). The global crisis could also lead donors to scale back their support. To deal with that possibility, the program includes target adjusters (see the Technical Memorandum of Understanding (TMU)).

\section{StafF APPRAISAL}

30. In a difficult postconflict environment, Burundi has made steady, though uneven, progress. Real GDP accelerated in 2008, though the food and fuel shock has kept inflation high. Most monetary and fiscal reforms have progressed well. The economic outlook is generally positive but subject to risks arising from the security situation and the external environment.

\section{The revised program for 2009 should further consolidate macroeconomic} stability and reduce poverty while helping Burundi cope with the impact of the global financial crisis. Staff agrees that easing fiscal and monetary policy in the near term is an appropriate policy response to the crisis. Spending on MDG-related sectors will be boosted significantly, supported by HIPC and MDRI debt relief.

32. The authorities should continue to rely mainly on grants and highly concessional loans to avoid unsustainable debt. Improvements in governance will be critical for donor support. To this end, the authorities should further strengthen PFM. Staff urges donors to accelerate their grant and other concessional support given the need to reduce poverty and mitigate the risk of debt distress.

33. In light of the global financial crisis, staff urges the authorities to accelerate their efforts to strengthen the financial sector by improving banking supervision, addressing weaknesses in the banking system, and enhancing central bank internal controls. The forthcoming Bank-Fund FSAP report should help guide these efforts. 
34. Sustained growth depends on accelerating structural reforms. Besides sustaining the momentum on fiscal governance reforms, the authorities should continue their efforts to reform the coffee sector. EAC membership should also spur structural reforms that would improve the business environment.

35. As a postconflict country, Burundi still faces significant risks, but so far the authorities have demonstrated their ability to manage economic and social pressures. Staff fully endorses their efforts.

36. Staff recommends completion of the second PRGF review, based on Burundi's performance and the strength of the program, and modification of performance criteria. 
Table 1. Burundi: Millennium Development Goals

\begin{tabular}{|c|c|c|c|c|c|c|c|}
\hline & 1990 & 1995 & 2001 & 2003 & 2004 & 2005 & $\begin{array}{r}2015 \\
\text { Target } \\
\end{array}$ \\
\hline \multicolumn{8}{|l|}{ Goal 1. Eradicate extreme poverty and hunger } \\
\hline \multicolumn{8}{|l|}{$\begin{array}{l}\text { Target 1: Halve between } 1990 \text { and } 2015 \text {, the proportion of people whose income is less } \\
\text { than US } \$ 1 \text { a day. }\end{array}$} \\
\hline 1. Population below US $\$ 1$ a day (percent) & $\ldots$ & $\ldots$ & $58.4^{1}$ & $\ldots$ & $\ldots$ & $\ldots$ & $\ldots$ \\
\hline 2. Poverty gap ratio at US\$1 a day (percent) & $\ldots$ & $\ldots$ & $22.7^{1}$ & $\ldots$ & $\ldots$ & $\ldots$ & $\ldots$ \\
\hline 3. Share of income or consumption held by poorest 20 percent (percent) & $7.9^{2}$ & $\ldots$ & $5.1^{1}$ & $\ldots$ & $\ldots$ & $\ldots$ & $\ldots$ \\
\hline \multicolumn{8}{|l|}{ Target 2: Halve between 1990 and 2015 the proportion of people suffering hunger } \\
\hline 4. Prevalence of child malnutrition (percent of children under 5) & $\ldots$ & $\ldots$ & $45.1^{3}$ & $\ldots$ & $\ldots$ & $\ldots$ & $\ldots$ \\
\hline 5. Population below minimum level of dietary energy consumption (percent) & 49.0 & 64.0 & 70.0 & 67.0 & $\ldots$ & 66.0 & 24.5 \\
\hline \multicolumn{8}{|l|}{ Goal 2. Achieve universal primary education } \\
\hline \multicolumn{8}{|l|}{$\begin{array}{l}\text { Target 3: Ensure that, by } 2015 \text {, children will be able to complete a full course of primary } \\
\text { schooling. }\end{array}$} \\
\hline 6. Net primary enrollment ratio (percent of relevant age group) & 41.0 & 29.5 & 52.3 & 30.6 & $\ldots$ & 35.7 & 100.0 \\
\hline 7. Percentage of cohort reaching grade 5 & 61.7 & $\ldots$ & 64.0 & 63.0 & $\ldots$ & 67.0 & 100.0 \\
\hline 8. Youth literacy rate (percent age 15-24) & 51.6 & 57.9 & 65.1 & $\ldots$ & 73.3 & $\ldots$ & 100.0 \\
\hline \multicolumn{8}{|l|}{ Goal 3. Promote gender equality and empower women } \\
\hline \multicolumn{8}{|l|}{$\begin{array}{l}\text { Target 4: Eliminate gender disparity in primary and secondary education preferably by } \\
2005 \text { and to all levels of education by } 2015 \text {. }\end{array}$} \\
\hline 9. Ratio of girls to boys in primary and secondary education (percent) & 81.8 & $\ldots$ & $\ldots$ & 79.1 & 82.0 & 82.8 & $\ldots$ \\
\hline 10. Ratio of young literate females to males (percent ages $15-24$ ) & 76.7 & 86.0 & 95.6 & $\ldots$ & 91.7 & $\ldots$ & $\ldots$ \\
\hline 11. Share of women employed in the nonagricultural sector (percent) & 13.3 & $\ldots$ & $\ldots$ & $\ldots$ & $\ldots$ & $\ldots$ & $\ldots$ \\
\hline 12. Proportion of seats held by women in the national parliament (percent) & $\ldots$ & 11.3 & 14.0 & 18.0 & 18.0 & 30.5 & $\ldots$ \\
\hline \multicolumn{8}{|l|}{ Goal 4. Reduce child mortality } \\
\hline \multicolumn{8}{|l|}{ Target 5: Reduce by two-thirds between 1990 and 2015 the under- 5 mortality rate } \\
\hline 13. Under-5 mortality rate (per 1,000$)$ & 190.0 & 190.0 & 204.0 & $\ldots$ & 190.0 & 190.0 & 63.3 \\
\hline 14. Infant mortality rate (per 1,000 live births) & 114.0 & 114.0 & 121.0 & $\ldots$ & 114.0 & 114.0 & $\ldots$ \\
\hline 15. Immunization against measles (percent of children $12-23$ months) & 74.0 & 80.0 & 75.0 & 75.0 & 75.0 & 75.0 & $\ldots$ \\
\hline \multicolumn{8}{|l|}{ Goal 5. Improve maternal health } \\
\hline \multicolumn{8}{|l|}{ Target 6: Reduce by three-quarters, between 1990 and 2015 , the maternal mortality ratio. } \\
\hline 16. Maternal mortality ratio (modeled estimate, per 100,000 live births) & $\ldots$ & $\ldots$ & $1,000.0^{3}$ & $\ldots$ & $\ldots$ & $\ldots$ & $\ldots$ \\
\hline 17. Proportion of births attended by skilled health personnel (precent of total) & $\ldots$ & $\ldots$ & $25.2^{3}$ & $\ldots$ & $\ldots$ & $\ldots$ & $\ldots$ \\
\hline \multicolumn{8}{|l|}{ Goal 6. Combat HIV/AIDS, malaria and other diseases } \\
\hline \multicolumn{8}{|l|}{ Target 7: Halt by 2015 , and begin to reverse, the spread of HIVIAIDS. } \\
\hline 18. HIV prevalence among females (percent ages $15-24$ ) & $\ldots$ & $\ldots$ & 11.0 & $\ldots$ & $\ldots$ & 2.3 & $\ldots$ \\
\hline 19. Contraceptive prevalence rate (percent of women ages 15-49) & $\ldots$ & $\ldots$ & $16.0^{3}$ & $\ldots$ & $\ldots$ & & $\ldots$ \\
\hline 20. Number of children orphaned by HIVIAIDS & $\ldots$ & $\ldots$ & 170,000 & 120,000 & $\ldots$ & 120,000 & $\ldots$ \\
\hline \multicolumn{8}{|l|}{$\begin{array}{l}\text { Target } 8 \text { : Halt by } 2015 \text {, and begin to reverse, the incidence of malaria and other major } \\
\text { diseases. }\end{array}$} \\
\hline 21. Prevalence of death associated with malaria & $\ldots$ & $\ldots$ & $\ldots$ & $\ldots$ & $\ldots$ & $\ldots$ & $\ldots$ \\
\hline 22. Share of population in malaria risk areas using effective prevention and treatment & $\ldots$ & $\ldots$ & $\ldots$ & $\ldots$ & $\ldots$ & $\ldots$ & $\ldots$ \\
\hline 23. Incidence of tuberculosis (per 100,000 people) & 124.6 & $\ldots$ & 298.3 & 334.2 & $\ldots$ & 333.7 & $\ldots$ \\
\hline 24. Tuberculosis cases detected by DOTS (percent) & $\ldots$ & 19.6 & 34.4 & 30.0 & 28.9 & 29.6 & $\ldots$ \\
\hline \multicolumn{8}{|l|}{ Goal 7. Ensure environmental sustainability } \\
\hline Target 9: Integrate the principles of sustainable development into policies and programs & & & & & & & \\
\hline Reverse the loss of environmental resources. & & & & & & & \\
\hline 25. Forest area (percent of total land area) & 11.3 & $\ldots$ & $\ldots$ & $\ldots$ & $\ldots$ & 5.9 & $\ldots$ \\
\hline 26. Nationally protected areas (percent of total land area) & 4.4 & 4.8 & 5.6 & 5.6 & 5.6 & 5.7 & $\ldots$ \\
\hline 27. GDP per unit of energy use (PPP US\$ per kg oil equivalent) & $\ldots$ & $\ldots$ & $\cdots$ & & $\ldots$ & $\ldots$ & $\ldots$ \\
\hline 28. $\mathrm{CO}_{2}$ emissions (metric tons per capita) & 0.03 & 0.04 & 0.04 & 0.03 & $\ldots$ & $\ldots$ & $\ldots$ \\
\hline 29. Proportion of population using solid fuels & & & & & & & \\
\hline Target 10: Halve by 2015 proportion of people without access to safe drinking water. & & & & & & & \\
\hline 30. Access to improved water source (percent of population) & 69.0 & $\ldots$ & $\ldots$ & $\ldots$ & 79.0 & 79.0 & 84.5 \\
\hline $\begin{array}{l}\text { Target 11: Achieve by } 2020 \text { significant improvement for at least } 100 \text { million slum dwelle } \\
\text { worldwide. }\end{array}$ & & & & & & & \\
\hline 31. Access to improved sanitation (percent of population) & 44.0 & $\ldots$ & $\ldots$ & $\ldots$ & 36.0 & 36.0 & $\ldots$ \\
\hline 32. Access to secure tenure (percent of population) & $\ldots$ & $\ldots$ & $\ldots$ & $\ldots$ & $\ldots$ & $\ldots$ & $\ldots$ \\
\hline Goal 8. Develop a Global Partnership for Development ${ }^{4 /}$ & & & & & & & \\
\hline Target 16: Develop and implement strategies for productive work for youth. & & & & & & & \\
\hline 45. Unemployment rate of population ages $15-24$ (total) & $\ldots$ & $\ldots$ & $\ldots$ & $\ldots$ & $\ldots$ & $\ldots$ & $\ldots$ \\
\hline Female & $\ldots$ & $\ldots$ & $\ldots$ & $\ldots$ & $\ldots$ & $\ldots$ & $\ldots$ \\
\hline Male & $\ldots$ & $\ldots$ & $\ldots$ & $\ldots$ & $\ldots$ & $\ldots$ & $\ldots$ \\
\hline Target 17: Provide access to affordable essential drugs. & & & & & & & \\
\hline 46. Proportion of population with access to affordable essential drugs & $\cdots$ & $\cdots$ & $\ldots$ & $\ldots$ & $\cdots$ & $\ldots$ & $\ldots$ \\
\hline $\begin{array}{l}\text { Target 18: Make available new technologies, especially information and communicatio } \\
47 \text {. Fixed line and mobile telephones (per } 1,000 \text { people) }\end{array}$ & & & & & & & \\
\hline $\begin{array}{l}\text { 47. Fixed line and mobile telephones (per } 1,000 \text { people) } \\
\text { 48. Personal computers (per } 1,000 \text { people) }\end{array}$ & $\begin{array}{r}1.4 \\
\ldots\end{array}$ & $\begin{array}{r}2.9 \\
\ldots\end{array}$ & $\begin{array}{l}7.4 \\
0.7\end{array}$ & $\begin{array}{r}12.5 \\
1.8\end{array}$ & $\begin{array}{r}12.5 \\
4.8\end{array}$ & $\begin{array}{r}17.6 \\
4.7\end{array}$ & $\begin{array}{l}\cdots \\
\cdots\end{array}$ \\
\hline
\end{tabular}

Sources: World Bank; World Development Indicators database, April 2006; and IMF staff estimates.

${ }^{1}$ Survey data for 1998.

${ }^{2}$ Survey data for 1992.

${ }^{3}$ Survey data for 2000 .

${ }^{4}$ Targets $12-15$ and indicators 33-44 are excluded because they cannot be measured on a country-specific basis. These are related to official development assistance, market access, and the HIPC Initiative. 
Table 2. Burundi: Selected Economic and Financial Indicators, 2007-12

\begin{tabular}{|c|c|c|c|c|c|c|c|c|}
\hline & \multirow{2}{*}{$\begin{array}{c}2007 \\
\text { Est. }\end{array}$} & \multicolumn{2}{|c|}{2008} & \multicolumn{2}{|c|}{2009} & \multirow[t]{2}{*}{2010} & \multirow{2}{*}{$\begin{array}{l}2011 \\
\text { Proj. }\end{array}$} & \multirow[t]{2}{*}{2012} \\
\hline & & Prog. & Prel. & Prog. & $\begin{array}{l}\text { Rev. } \\
\text { Prog. }\end{array}$ & & & \\
\hline & \multicolumn{8}{|c|}{ (Annual percentage change) } \\
\hline National income and prices & & & & & & & & \\
\hline Real GDP growth & 3.6 & 4.5 & 4.5 & 4.5 & 3.2 & 3.6 & 4.2 & 4.8 \\
\hline GDP deflator & 8.2 & 18.5 & 24.4 & 16.5 & 20.3 & 6.8 & 6.0 & 5.4 \\
\hline Consumer prices (period average) & 8.3 & 19.1 & 24.4 & 11.2 & 12.9 & 8.3 & 6.8 & 5.8 \\
\hline Consumer prices (end of period) & 14.7 & 14.0 & 25.7 & 9.0 & 9.1 & 7.5 & 6.0 & 5.5 \\
\hline \multicolumn{9}{|l|}{ External sector } \\
\hline Exports, f.o.b. (US\$) & -9.7 & 49.7 & 9.0 & -11.5 & -0.4 & 27.1 & 7.3 & 3.6 \\
\hline Imports, f.o.b. (US\$) & 20.6 & 18.4 & 16.0 & -18.0 & -32.3 & 16.7 & 12.1 & 9.1 \\
\hline Export volume & -1.7 & 34.0 & -0.4 & -0.8 & 35.5 & 27.2 & 6.6 & 4.2 \\
\hline Import volume & 6.0 & 4.3 & 4.2 & 7.5 & 6.0 & 6.1 & 5.5 & 5.4 \\
\hline Terms of trade (deterioration $=-$ ) & -23.4 & 3.7 & 3.5 & 16.8 & 15.0 & -9.2 & -5.3 & -4.0 \\
\hline & \multicolumn{8}{|c|}{$\begin{array}{l}\text { (Change in percent of beginning of period } \mathrm{M} 2 \text {, } \\
\text { unless otherwise indicated) }\end{array}$} \\
\hline Money and credit & & & & & & & & \\
\hline Net foreign assets & 11.5 & 12.5 & 21.9 & -6.6 & 0.3 & $\ldots$ & $\ldots$ & $\ldots$ \\
\hline Domestic credit & 6.6 & 21.0 & 18.8 & 21.9 & 15.7 & $\ldots$ & $\ldots$ & $\ldots$ \\
\hline Government & -0.2 & 0.0 & 5.8 & 0.0 & 4.9 & $\ldots$ & $\ldots$ & $\ldots$ \\
\hline Private sector & 7.6 & 16.6 & 8.5 & 18.4 & 6.2 & $\ldots$ & $\ldots$ & $\ldots$ \\
\hline Money and quasi money (M2) & 10.1 & 20.0 & 34.2 & 13.9 & 14.4 & $\ldots$ & $\ldots$ & $\ldots$ \\
\hline \multirow[t]{2}{*}{ Reserve money (12-month growth rate) } & 17.4 & 22.0 & 25.1 & 12.9 & 13.5 & $\cdots$ & $\cdots$ & $\cdots$ \\
\hline & \multicolumn{8}{|c|}{ (Percent of GDP) } \\
\hline General government & & & & & & & & \\
\hline Revenue (excluding grants) & 18.6 & 19.1 & 18.6 & 19.3 & 18.0 & 18.6 & 19.3 & 19.5 \\
\hline Total expenditure and net lending & 38.5 & 42.6 & 44.1 & 44.6 & 46.1 & 45.5 & 41.8 & 41.2 \\
\hline \multicolumn{9}{|l|}{ Overall balance (commitment basis) } \\
\hline Excluding grants & -19.8 & -23.5 & -25.5 & -25.4 & -28.1 & -26.9 & -22.5 & -21.7 \\
\hline Including grants (excl. HIPC) & -3.3 & -3.6 & -3.8 & -3.0 & -4.1 & -4.0 & -1.9 & -1.3 \\
\hline Savings-Investment balance & -15.7 & -18.2 & -14.2 & -11.5 & -9.5 & -10.6 & -13.6 & -14.7 \\
\hline Private & -12.4 & -17.7 & -10.5 & -8.5 & -5.4 & -6.7 & -11.7 & -13.4 \\
\hline Public & -3.3 & -0.5 & -3.8 & -3.0 & -4.1 & -4.0 & -1.9 & -1.3 \\
\hline & \multicolumn{8}{|c|}{ (US\$ million, unless otherwise indicated) } \\
\hline External sector & & & & & & & & \\
\hline $\begin{array}{l}\text { Current account, including grants } \\
\text { Gross official reserves }\end{array}$ & -153.4 & -207.1 & -155.9 & -163.3 & -134.6 & -153.7 & -206.0 & -236.3 \\
\hline In US\$ millions & 177.3 & 155.3 & 266.6 & 153.8 & 231.8 & 247.7 & 249.8 & 252.1 \\
\hline In months of imports & 3.9 & 3.2 & 6.6 & 3.4 & 5.5 & 5.0 & 4.5 & 4.0 \\
\hline Debt-service to exports ratio (in percent) & 6.8 & 4.3 & 3.6 & 1.6 & 2.3 & 3.4 & 3.8 & 6.3 \\
\hline \multicolumn{9}{|l|}{ Memorandum item: } \\
\hline GDP at current market prices (Fbu billion) & 1,060 & 1,311 & 1,378 & 1,678 & 1,711 & 1,893 & 2,091 & 2,310 \\
\hline
\end{tabular}

Sources: Burundi authorities; and IMF staff estimates and projections. 
Table 3. Burundi: General Government Operations, 2007-12

\begin{tabular}{|c|c|c|c|c|c|c|c|c|}
\hline & \multirow{2}{*}{$\begin{array}{r}2007 \\
\text { Est. }\end{array}$} & \multicolumn{2}{|l|}{2008} & \multicolumn{2}{|c|}{2009} & 2010 & 2011 & 2012 \\
\hline & & Prog. & Est. & Prog. & $\begin{array}{l}\text { Rev. } \\
\text { Prog. }\end{array}$ & \multicolumn{3}{|c|}{ Proj. } \\
\hline & \multicolumn{8}{|c|}{ (Percent of GDP) } \\
\hline Revenue & 18.6 & 19.1 & 18.6 & 19.3 & 18.0 & 18.6 & 19.3 & 19.5 \\
\hline Tax revenue & 17.2 & 17.7 & 16.7 & 17.4 & 16.3 & 16.8 & 17.5 & 17.7 \\
\hline Income tax & 5.0 & 4.6 & 4.7 & 4.8 & 4.5 & 4.6 & 4.8 & 4.9 \\
\hline Taxes on goods and services & 8.7 & 9.5 & 8.8 & 8.7 & 8.4 & 8.8 & 8.9 & 8.8 \\
\hline Taxes on international trade & 3.2 & 3.3 & 3.0 & 3.7 & 3.1 & 3.2 & 3.5 & 3.7 \\
\hline Other tax revenue & 0.3 & 0.3 & 0.2 & 0.3 & 0.3 & 0.3 & 0.3 & 0.3 \\
\hline Nontax revenue & 1.4 & 1.4 & 2.0 & 1.8 & 1.7 & 1.8 & 1.8 & 1.8 \\
\hline Expenditure and net lending & 38.5 & 42.6 & 44.1 & 44.6 & 46.1 & 45.5 & 41.8 & 41.2 \\
\hline Current expenditure ${ }^{1}$ & 24.6 & 25.2 & 25.7 & 24.5 & 24.6 & 25.3 & 25.4 & 25.6 \\
\hline of which: Salaries & 10.8 & 10.8 & 11.2 & 10.7 & 11.2 & 11.1 & 10.8 & 10.7 \\
\hline Externally financed special programs ${ }^{2}$ & 1.2 & 1.8 & 3.2 & 3.8 & 5.3 & 3.0 & 0.9 & 0.8 \\
\hline $\begin{array}{l}\text { Capital expenditure \& Net lending } \\
\text { of which: MDRI related }\end{array}$ & 12.7 & 15.6 & 15.2 & $\begin{array}{r}16.3 \\
1.7\end{array}$ & $\begin{array}{r}16.3 \\
2.3\end{array}$ & $\begin{array}{r}17.2 \\
1.0\end{array}$ & 15.6 & 14.8 \\
\hline Overall balance (commitment basis) & -19.8 & -23.5 & -25.5 & -25.4 & -28.1 & -26.9 & -22.5 & -21.7 \\
\hline (after grants, excl. HIPC) & -3.3 & -3.6 & -3.8 & -3.0 & -4.1 & -4.0 & -1.9 & -1.3 \\
\hline Change in arrears (reduction $=-$ ) & -2.1 & -1.3 & -0.8 & 0.0 & -0.2 & 0.0 & 0.0 & 0.0 \\
\hline $\begin{array}{l}\text { Overall balance (cash basis) } \\
\text { (after grants, excl. HIPC) }\end{array}$ & $\begin{array}{r}-21.9 \\
-5.4\end{array}$ & $\begin{array}{r}-24.8 \\
-4.9\end{array}$ & $\begin{array}{r}-26.3 \\
-4.6\end{array}$ & $\begin{array}{r}-25.4 \\
-3.0\end{array}$ & $\begin{array}{r}-28.3 \\
-4.3\end{array}$ & $\begin{array}{r}-26.9 \\
-4.0\end{array}$ & $\begin{array}{r}-22.5 \\
-1.9\end{array}$ & $\begin{array}{r}-21.7 \\
-1.3\end{array}$ \\
\hline Financing & 22.4 & 24.8 & 26.6 & 25.4 & 28.3 & 25.9 & 21.5 & 20.7 \\
\hline External grants & 20.9 & 23.0 & 25.0 & 86.1 & 86.5 & 22.0 & 19.7 & 19.5 \\
\hline of which: HIPC relief & 3.8 & 3.1 & 3.0 & 63.8 & 62.5 & 0.0 & 0.0 & 0.0 \\
\hline MDRI grant from IMF & 0.0 & 0.0 & 0.0 & 1.7 & 2.3 & 1.0 & 0.0 & 0.0 \\
\hline External borrowing, net & -0.9 & 0.7 & 0.2 & -60.9 & -59.6 & 2.6 & 3.0 & 1.8 \\
\hline Privatization proceeds & 0.0 & 0.1 & 0.0 & 0.1 & 0.1 & 0.1 & 0.1 & 0.1 \\
\hline Domestic & 2.4 & 1.0 & 1.4 & 0.0 & 1.3 & 1.2 & -1.2 & -0.7 \\
\hline \multirow[t]{2}{*}{ Financing gap and errors and omissions ${ }^{3}$} & -0.5 & 0.0 & -0.3 & 0.0 & 0.0 & 1.0 & 1.0 & 1.0 \\
\hline & \multicolumn{8}{|c|}{ (Fbu billion) } \\
\hline Total Revenue & 197.6 & 249.8 & 256.7 & 323.3 & 308.1 & 352.1 & 402.8 & 449.3 \\
\hline Total Expenditure and net lending & 407.9 & 558.0 & 608.0 & 748.8 & 789.6 & 861.3 & 873.3 & 951.0 \\
\hline Financing gap and errors and omissions ${ }^{3}$ & -5.4 & 0.0 & -4.5 & 0.0 & 0.0 & 18.5 & 21.0 & 23.0 \\
\hline Total Financing & 237.7 & 325.7 & 366.8 & 425.6 & 485.1 & 490.8 & 449.5 & 478.7 \\
\hline & \multicolumn{8}{|c|}{ (USD million, unless otherwise indicated) } \\
\hline \multicolumn{9}{|l|}{ Memorandum items: } \\
\hline MDRI stock relief from IDA and AfDB & & & & 84.8 & 84.8 & & & \\
\hline \multicolumn{9}{|l|}{ MDRI savings from IDA and AfDB: } \\
\hline Ammortization & & & & 2.3 & 2.3 & 3.0 & 3.2 & 3.3 \\
\hline Annual interest payments & & & & 0.5 & 0.5 & 0.6 & 0.6 & 0.6 \\
\hline Social expenditure (percent of GDP) & 9.2 & 8.2 & 8.8 & 9.9 & 9.9 & 10.3 & 11.2 & 11.0 \\
\hline Public debt (percent of GDP) & 178 & 142 & 154 & 44 & 49 & 50 & 49 & 46 \\
\hline GDP at current market prices (Fbu billions) & 1060 & 1311 & 1378 & 1678 & 1711 & 1893 & 2091 & 2310 \\
\hline
\end{tabular}

Sources: Burundi authorities, and IMF staff estimates and projections.

${ }^{1}$ The medium-term current spending projections are mostly driven by health, education, food security, and agriculture expenditures.

${ }^{2}$ These are externally financed expenditures, and include spending on elections, demobilization, and one-off temporary social safety net programs.

${ }^{3}$ Expected to be financed by additional donor support. 
Table 4. Burundi: Monetary Survey, 2005-09

\begin{tabular}{|c|c|c|c|c|c|c|c|}
\hline & \multirow[t]{2}{*}{2005} & \multirow{2}{*}{$\frac{2006}{\text { Actual }}$} & \multirow[t]{2}{*}{2007} & \multicolumn{2}{|c|}{2008} & \multicolumn{2}{|c|}{2009} \\
\hline & & & & Prog. & Est. & Prog. & Rev. \\
\hline & \multicolumn{7}{|c|}{ (Fbu billion) } \\
\hline Net foreign assets & 70.3 & 74.6 & 109.1 & 150.3 & 181.5 & 124.0 & 182.9 \\
\hline Central bank & 48.8 & 41.5 & 75.6 & 38.7 & 139.4 & 25.0 & 87.3 \\
\hline Deposit money banks & 21.5 & 33.1 & 33.5 & 111.5 & 42.0 & 98.9 & 95.6 \\
\hline Net domestic assets & 227.6 & 277.7 & 298.7 & 390.5 & 368.9 & 477.4 & 438.9 \\
\hline Domestic credit & 277.9 & 354.3 & 374.1 & 443.3 & 436.1 & 529.9 & 505.5 \\
\hline Net claims on the government & 121.1 & 158.1 & 157.4 & 157.4 & 176.5 & 157.3 & 198.1 \\
\hline Credit to the economy & 156.8 & 196.2 & 216.6 & 286.0 & 259.7 & 372.6 & 307.4 \\
\hline Other items, net (assets $=+$ ) & -50.4 & -76.6 & -75.4 & -52.9 & -67.2 & -52.5 & -66.6 \\
\hline M3 & 297.8 & 352.2 & 407.8 & 540.8 & 550.4 & 601.4 & 621.9 \\
\hline Foreign currency deposits & 40.2 & 52.3 & 77.7 & 144.5 & 107.3 & 150.0 & 115.0 \\
\hline M2 & 257.7 & 299.9 & 330.1 & 396.2 & 443.1 & 451.4 & 506.9 \\
\hline Currency in circulation & 67.9 & 68.4 & 84.2 & 117.6 & 112.6 & 135.2 & 135.2 \\
\hline Local currency deposits & 189.8 & 231.5 & 245.9 & 278.6 & 330.5 & 316.2 & 371.7 \\
\hline Demand deposits & 115.6 & 147.5 & 151.2 & 186.4 & 223.3 & 211.6 & 244.7 \\
\hline \multirow[t]{2}{*}{ Quasi-money } & 74.2 & 84.0 & 94.7 & 92.2 & 107.2 & 104.7 & 127.0 \\
\hline & \multicolumn{7}{|c|}{ (Change as percent of beginning of period M2) } \\
\hline Net foreign assets & 15.9 & 1.7 & 11.5 & 12.5 & 21.9 & -6.6 & 0.3 \\
\hline Central bank & 13.8 & -2.8 & 11.4 & -11.2 & 19.3 & -3.5 & -11.7 \\
\hline Deposit money banks & 2.1 & 4.5 & 0.1 & 23.6 & 2.6 & -3.2 & 12.1 \\
\hline Net domestic assets & 8.6 & 19.4 & 7.0 & 27.8 & 21.3 & 21.9 & 15.8 \\
\hline Domestic credit & -0.4 & 29.6 & 6.6 & 21.0 & 18.8 & 21.9 & 15.7 \\
\hline Net claims on the government & 7.2 & 14.4 & -0.2 & 0.0 & 5.8 & 0.0 & 4.9 \\
\hline Credit to the economy & -7.6 & 15.3 & 6.8 & 21.0 & 13.0 & 21.9 & 10.8 \\
\hline of which: private sector & -6.7 & 13.7 & 7.6 & 16.6 & 8.5 & 18.4 & 6.2 \\
\hline Other items, net (assets $=+$ ) & 9.0 & 13.7 & 0.4 & 6.8 & 2.5 & 0.1 & 0.1 \\
\hline M3 & 24.5 & 21.1 & 18.5 & 40.3 & 43.2 & 15.3 & 16.1 \\
\hline Foreign currency deposits & -2.6 & 4.7 & 8.5 & 20.2 & 9.0 & 1.4 & 1.7 \\
\hline M2 & 27.1 & 16.4 & 10.1 & 20.0 & 34.2 & 13.9 & 14.4 \\
\hline Currency in circulation & 5.3 & 0.2 & 5.2 & 10.1 & 8.6 & 4.4 & 5.1 \\
\hline Local currency deposits & 21.8 & 16.2 & 4.8 & 9.9 & 25.6 & 9.5 & 9.3 \\
\hline Demand deposits & 7.7 & 12.4 & 1.2 & 10.7 & 21.8 & 6.4 & 4.8 \\
\hline Quasi-money & 14.1 & 3.8 & 3.6 & -0.8 & 3.8 & 3.1 & 4.5 \\
\hline \multicolumn{8}{|l|}{ Memorandum items: } \\
\hline Reserve money (12-month percent change) & 32.7 & 5.9 & 17.4 & 22.0 & 25.1 & 12.9 & 13.5 \\
\hline
\end{tabular}

Sources: Banque de la République du Burundi (BRB); and IMF staff estimates and projections. 
Table 5. Burundi: Central Bank Accounts, 2006-09

\begin{tabular}{|c|c|c|c|c|c|c|c|c|c|c|c|c|c|}
\hline & \multirow{3}{*}{$\begin{array}{l}2006 \\
\text { Dec. }\end{array}$} & \multicolumn{4}{|c|}{2007} & \multicolumn{4}{|c|}{2008} & \multicolumn{4}{|c|}{2009} \\
\hline & & \multirow[t]{2}{*}{ Mar. } & \multirow[t]{2}{*}{ Jun. } & \multirow[t]{2}{*}{ Sept. } & \multirow[t]{2}{*}{$\overline{\text { Dec. }}$} & \multirow[t]{2}{*}{ Mar. } & \multirow[t]{2}{*}{ Jun. } & \multirow[t]{2}{*}{ Sept. } & \multirow[t]{2}{*}{$\overline{\text { Dec. }}$} & \multirow[t]{2}{*}{ Mar. } & Jun. & Sept. & Dec. \\
\hline & & & & & & & & & & & \multicolumn{2}{|c|}{ Rev. Prog. } & \\
\hline & \multicolumn{13}{|c|}{ (Fbu billion) } \\
\hline Net foreign assets & 41.5 & 30.9 & 17.8 & 5.8 & 75.6 & 69.9 & 63.4 & 75.1 & 139.4 & 105.1 & 42.1 & 48.4 & 87.3 \\
\hline Net domestic assets & 65.0 & 68.9 & 91.1 & 104.9 & 49.4 & 48.4 & 71.6 & 71.4 & 17.0 & 33.6 & 122.6 & 127.4 & 90.2 \\
\hline Domestic credit & 165.3 & 125.1 & 143.5 & 151.4 & 138.8 & 125.0 & 156.3 & 146.9 & 114.1 & 108.0 & 193.7 & 200.7 & 173.8 \\
\hline Net claims on the government & 161.4 & 120.4 & 140.3 & 148.1 & 134.7 & 121.6 & 150.7 & 141.4 & 120.8 & 110.3 & 117.7 & 125.1 & 132.6 \\
\hline Nongovernment credit & 3.9 & 4.7 & 4.6 & 3.0 & 3.2 & 3.4 & 5.6 & 5.4 & -6.6 & -2.3 & 76.0 & 75.6 & 41.2 \\
\hline Other items, net (assets $=+$ ) & -100.2 & -56.1 & -52.4 & -46.5 & -89.4 & -80.1 & -78.3 & -75.6 & -97.2 & -74.4 & -71.1 & -73.4 & -83.6 \\
\hline Reserve money & 106.5 & 99.8 & 108.9 & 110.8 & 125.0 & 118.3 & 135.1 & 146.5 & 156.4 & 138.7 & 164.7 & 175.8 & 177.5 \\
\hline Currency in circulation & 68.4 & 62.9 & 71.7 & 78.7 & 84.2 & 80.6 & 99.3 & 110.4 & 112.6 & 99.9 & 125.2 & 133.3 & 135.2 \\
\hline Bank reserves & 28.2 & 26.6 & 27.5 & 21.8 & 24.9 & 23.5 & 18.9 & 17.3 & 25.0 & 22.2 & 23.1 & 26.0 & 25.9 \\
\hline Cash in vault & 8.4 & 7.7 & 7.9 & 8.0 & 8.8 & 9.1 & 9.9 & 12.6 & 11.6 & 12.7 & 12.7 & 12.7 & 12.7 \\
\hline Other nonbank deposits & 1.4 & 2.6 & 1.8 & 2.2 & 7.1 & 5.1 & 7.1 & 6.2 & 7.2 & 3.8 & 3.8 & 3.8 & 3.8 \\
\hline \multicolumn{14}{|l|}{ Memorandum items: } \\
\hline NFA of BRB (US\$ million) & 41.4 & 29.6 & 16.3 & 5.2 & 67.5 & 59.3 & 53.1 & 63.1 & 112.9 & 87.9 & 35.0 & 40.0 & 69.0 \\
\hline
\end{tabular}

Sources: Banque de la République du Burundi (BRB); and IMF staff estimates and projections. 
Table 6. Burundi: Balance of Payments, 2007-12

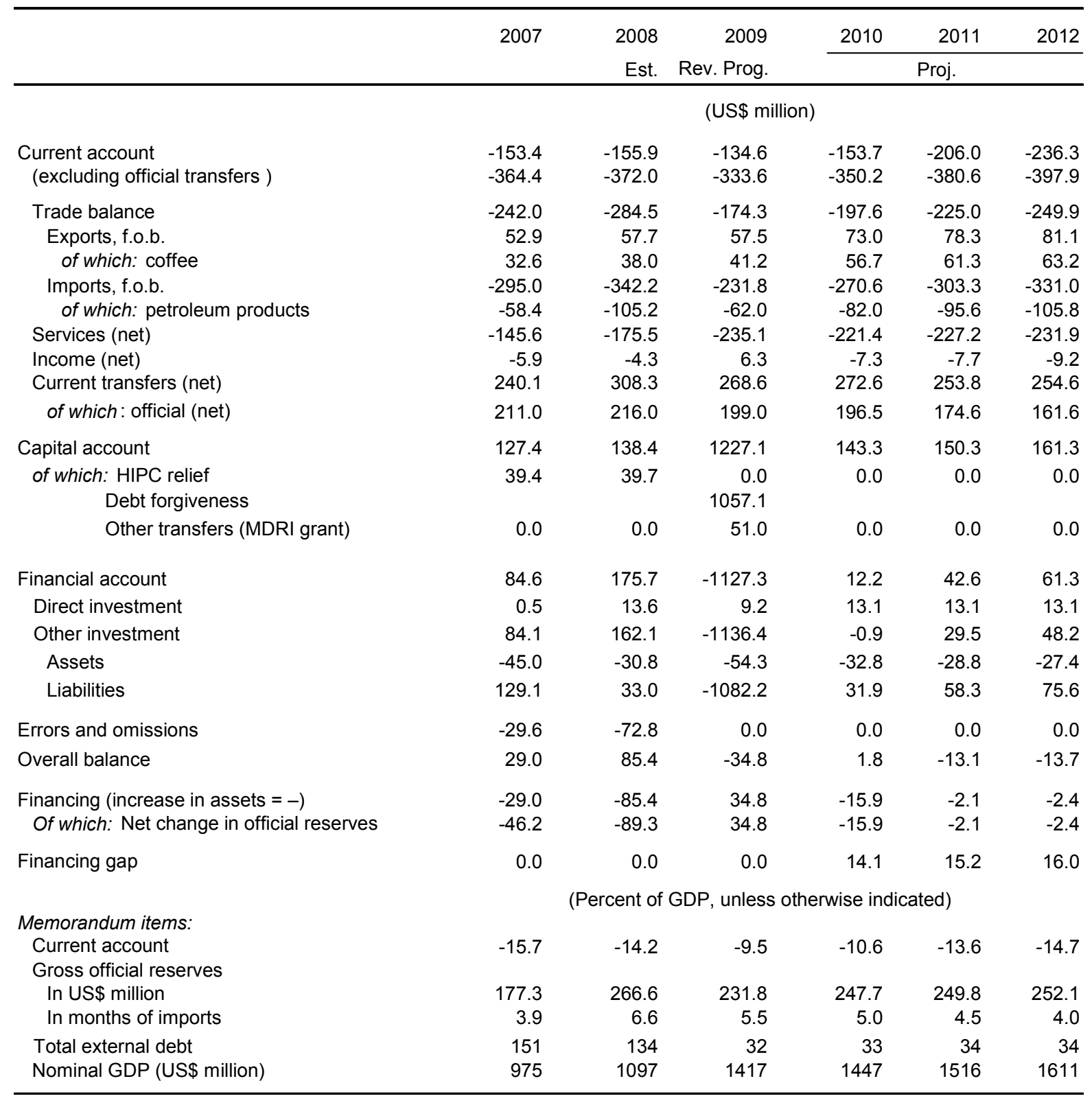

Sources: Burundi authorities; and IMF staff estimates and projections. 
Table 7. Burundi: Banking System Soundness Indicators, 2005-09

(in percent, unless otherwise indicated)

\begin{tabular}{|c|c|c|c|c|c|c|c|}
\hline & Dec-05 & Dec-06 & Dec-07 & Dec-08 & Jan-09 & Feb-09 & Mar-09 \\
\hline \multicolumn{8}{|l|}{ Capital Requirement } \\
\hline Capital requirement over weighted assets (solvency ratio) & 18.3 & 13.5 & 13.5 & 15.2 & 15.4 & 15.6 & 16.4 \\
\hline Core capital (Tier 1 capital) over weighted assets ${ }^{1}$ & 16.6 & 7.5 & 10.9 & 12.7 & 12.7 & 13.0 & 13.6 \\
\hline \multicolumn{8}{|l|}{ Quality of assets } \\
\hline Nonperforming loans (percent of total gross loans granted) & 20.6 & 18.6 & 18.8 & 14.6 & 16.2 & 16.1 & 15.4 \\
\hline Provisions (percent of nonperforming loans) & 83.0 & 92.4 & 91.4 & 90.2 & 89.7 & 88.9 & 90.0 \\
\hline Nonperforming loans net of provisions (percent of capital) & 20.4 & 7.5 & 7.4 & 7.4 & 7.9 & 8.1 & 6.9 \\
\hline Large exposures (percent of capital) & 34.3 & 48.6 & 41.6 & 39.3 & 37.0 & 39.5 & 32.5 \\
\hline \multicolumn{8}{|l|}{ Profitability rates } \\
\hline Return on assets & 1.9 & 1.7 & 2.3 & 2.3 & 0.2 & 0.7 & 1.1 \\
\hline Return on equity capital & 14.1 & 17.5 & 26.4 & 29.7 & 2.5 & 7.0 & 10.5 \\
\hline Net interest (percent of gross results) & 172.7 & 177.4 & 167.6 & 171.8 & 217.2 & 227.2 & 209.2 \\
\hline Costs excluding interest (percent of gross outturn) & 193.6 & 188.9 & 156.6 & 155.8 & 140.8 & 169.6 & 192.0 \\
\hline \multicolumn{8}{|l|}{ Liquidity } \\
\hline Liquid assets (percent of all loans granted) & 75.6 & 68.4 & 83.5 & 86.9 & 53.4 & 55.2 & 89.7 \\
\hline Liquid assets (percent of short-term commitments) & 87.0 & 106.8 & 135.2 & 137.9 & 157.3 & 161.5 & 153.9 \\
\hline
\end{tabular}

Sources: Burundian authorities; and IMF estimates.

${ }^{1}$ The decrease is due to the revision of Article 2 in Directive No. 2/06 of November 24, 2006. It is related to the calculation of basic equity capital, which no longer includes general provisions for risks. 
Table 8. Burundi: Actual and Projected Schedule of PRGF Disbursements and Reviews, 2008-11

\begin{tabular}{lcl}
\hline \multicolumn{1}{c}{ Date } & $\begin{array}{c}\text { Disbursement } \\
\text { (SDR million) }\end{array}$ & \multicolumn{1}{c}{ Conditions } \\
\hline $\begin{array}{l}\text { July 15, } 2008 \\
\text { February 9, } 2009\end{array}$ & 6.6 & $\begin{array}{l}\text { Executive Board approval } \\
\text { Completion of first review, based on } \\
\text { observance of performance criteria at } \\
\text { end-September 2008. }\end{array}$ \\
July 13, 2009 & 6.6 & $\begin{array}{l}\text { Completion of second review, based on } \\
\text { observance of performance criteria at } \\
\text { end-March 2009. }\end{array}$ \\
January 15, 2010 & 6.6 & $\begin{array}{l}\text { Completion of third review, based on } \\
\text { observance of performance criteria at } \\
\text { end-September 2009. }\end{array}$ \\
July 6, 2010 & 6.6 & $\begin{array}{l}\text { Completion of fourth review, based on } \\
\text { observance of performance criteria at } \\
\text { end-March 2010. }\end{array}$ \\
January 15, 2011 & 6.6 & $\begin{array}{l}\text { Completion of fifth review, based on } \\
\text { observance of performance criteria at } \\
\text { end-September 2010. }\end{array}$ \\
July 6, 2011 & & $\begin{array}{l}\text { Completion of sixth review, based on } \\
\text { observance of performance criteria at } \\
\text { end-March 2011. }\end{array}$ \\
\hline
\end{tabular}




\title{
APPENDIX I \\ TRANSLATED FROM FRENCH
}

\author{
BURUNDI \\ LETTER OF INTENT
}

Bujumbura, June 26, 2009

Mr. Dominique Strauss-Kahn

Managing Director

International Monetary Fund

Washington, D.C., 20431

Dear Mr. Strauss Kahn:

1. The Executive Board of the International Monetary Fund (IMF) approved a three-year arrangement under the Poverty Reduction and Growth Facility (PRGF) for the Republic of Burundi on July 7, 2008. This arrangement supports the medium-term program (April 1, 2008 to March 31, 2011) aimed at continuing the process of macroeconomic stabilization, reducing poverty, promoting structural reforms, and improving governance. In accordance with the terms of this arrangement, the government of Burundi carried out the second review of the program together with a mission from the IMF. This review focused on implementation of the program between October 1, 2008, and March 31, 2009, as well as the outlook and economic and financial measures to be implemented in 2009.

2. On the political front, the government of Burundi continues to make every effort to consolidate the peace process by implementing the agreements signed between Burundi and the warring parties. Moreover, the government is taking the necessary steps to organize the elections in 2010.

3. On the economic and social front, the government of Burundi is pleased to report that implementation of the program has been satisfactory, despite the difficult international situation resulting from the global financial crisis. In particular, all the quantitative and structural performance criteria for end-March 2009 have been met.

4. The government is resolved to continue implementing the policies and measures described in the Poverty Reduction Strategy Paper (PRSP). The Memorandum on Economic and Financial Policies (MEFP) attached to this letter completes the memorandums dated June 24, 2008, and January 8, 2009. 
5. The government believes that the policies set forth in the attached MEFP are adequate to achieve the objectives of its program. It will take any further measures that may become appropriate for this purpose. The Burundi authorities will consult with the IMF on the adoption of such measures and in advance of revisions to the policies contained in the MEFP, in accordance with the IMF's policies on such consultations.

6. The government of Burundi will provide the IMF with such information as it may request to monitor progress made in economic and financial policy implementation. Burundi will also carry out reviews of the PRGF-supported program with the IMF every six months. The third review should be completed no later than January 2010 and the fourth review no later than July 2010.

7. In view of the considerable progress made in implementing the program supported by the PRGF, the government is requesting completion of the second review and the third PRGF disbursement of SDR 6.6 million. The government is also requesting (1) modification of the performance criteria for end-September to take into account the impact of the global financial crisis; and (2) conversion of all structural performance criteria into benchmarks.

8. As in the past, the Burundi authorities wish to make this letter available to the public, along with the attached MEFP and Technical Memorandum of Understanding (TMU), as well as the IMF staff reports on the second PRGF review. We therefore authorize their publication and posting on the IMF website, subject to Executive Board approval. These documents will also be posted on the official websites of the Burundi government.

Sincerely yours,

Clotilde NIZIGAMA
Minister of Finance

\author{
$/ \mathrm{s} /$ \\ Gaspard SINDAYIGAYA \\ Governor, Bank of the Republic of Burundi
}

$\frac{/ \mathrm{s} /}{\text { Gabriel NTISEZERANA }} \overline{ }$
Second Vice President, Republic of Burundi

Attachments: $\quad$ Memorandum on Economic and Financial Policies

Technical Memorandum of Understanding 


\title{
APPENDIX I \\ ATtACHMENT I \\ TRANSLATED FROM FRENCH
}

\author{
BURUNDI \\ Memorandum on ECONOMic ANd Financial Policies
}

\section{INTRODUCTION}

1. This Memorandum on Economic and Financial Policies (MEFP) completes the memorandums dated June 24, 2008, and January 8, 2009. It provides an update on program implementation and the medium-term outlook and economic and financial policies that will be implemented in 2009 within the framework of the program covering April 1, 2008, to March 31, 2011. The measures and objectives contained in this MEFP are compatible with the Poverty Reduction Strategy Paper (PRSP) published in September 2006 and the findings of the annual PRSP implementation report sent to the IMF and the World Bank in November 2008.

2. Economic policy will continue to be guided by the following objectives: (1) return to single-digit inflation; (2) improve the composition of public spending to the benefit of priority sectors while preserving fiscal sustainability; (3) strengthen public financial management (PFM) and good governance; and (4) strengthen the internal control systems of the central bank.

3. With the continued improvement in the security situation, the macroeconomic objectives are as follows for the period of the PRGF: (1) GDP growth should average 4 percent over the medium term, compared to the 2004-07 average of 3.6 percent; (2) inflation should slow to about 6 percent in 2011; and (3) gross official reserves should stabilize at coverage of four months of imports.

\section{Program Implementation}

4. Macroeconomic developments have been generally in line with the program, although inflation was higher than anticipated. In 2008, real GDP growth accelerated to 4.5 percent, owing to a good coffee harvest. Inflation stood at 25.7 percent, compared to the targeted 14 percent, as a result of the increase in world food and oil prices. The overall fiscal deficit (cash basis, including non-HIPC grants) is estimated at about 4.6 percent of GDP, slightly below the target of 4.9 percent. Burundi's external position improved significantly, as reflected in the larger-than-anticipated increase in international reserves. 
5. All the quantitative performance criteria for end-March 2009 have largely been met. The ceilings on the wage bill and reserve money were met. However, the target for cumulative domestic arrears was not met because of the delay in disbursements of budgetary assistance.

6. In close collaboration with development partners, the government is strongly pursuing structural reform, particularly the promotion of transparency and good fiscal management, financial sector reform, coffee sector reform, oil sector reform, and regional integration. In addition to the achievements described in the previous memorandum dated January 8, 2009, significant progress has been made since then in all these areas.

7. In the area of public financial management (PFM), the government adopted the PFM strategy and action plan in the Council of Ministers, which will give new impetus to fiscal reform. The rationalization of government accounts is continuing without interfering with the smooth operation of the units concerned. To modernize the tax system, Parliament also adopted a law on the value-added tax (VAT), which will come into effect on July 1, 2009, in accordance with the commitments taken in the context of the East African Community (EAC). To better control the wage bill, the Ministry of Finance took over payroll management (end-March 2009 performance criterion). Moreover, the government also completed the census of civilian, military, and police employees. The report reveals that the status of 1,801 employees remains to be validated. Any ghost workers will be eliminated from the payroll once the validation is completed.

8. In the financial sector, the central bank continues to implement important measures to strengthen its internal control and risk management system, in line with the recommendations made in the recent safeguards assessment report prepared by IMF staff. In this context, an international auditor conducted special audits of the controls on large domestic transfers and disbursements to the government or its creditors in 2008.

9. In the monetary sector, the system of required reserves has been aligned with best practices, while the system of liquidity auctions was reformed with the removal of the ceiling on interest rates. In the area of foreign exchange, the Directives for Foreign Exchange Reserve Management were adopted and the Reserve Management Committee was reactivated. The central bank took measures to correct the dysfunctions in the foreign exchange auction market (MED), specifically by eliminating the floor price and the prior announcement of the amount to be auctioned. Moreover, it launched a symmetrical MED to make it possible to organize foreign exchange purchase auctions as well.

10. In the area of banking supervision, the decision to increase the minimum capital requirements for banks in 2009 was published. The central bank's (BRB) self-evaluation on compliance of its supervision with the Basel principles was completed, and an action plan for the implementation of corrective measures was prepared. In addition, reorganization of the banking supervision unit is proceeding well. 
11. An important step was taken in the reform of the coffee sector with the decree creating and setting out the bylaws of the Burundi Coffee Sector Regulatory Authority. Invitations to bid for the sale of coffee washing stations were published on June 5, 2009. As part of the oil sector reform, the government adopted a decree in May 2009 setting out the terms for monthly adjustment of retail prices for petroleum products on the basis of a World Bank study discussed with all participants in the sector. The government also benefited from technical assistance from the IMF's Fiscal Affairs Department on the use of fiscal and public spending policies to better protect the poor from the impact of such a price adjustment mechanism.

\section{ECONOMIC Prospects AND Policies for 2009}

12. The international financial crisis will have a negative impact on the economy of Burundi in 2009. GDP growth should slow and fall from 4.5 percent in 2008 to about 3.2 percent owing to (1) reduced demand for exports; (2) the decline in coffee prices; and (3) the likely fall in remittances and foreign direct investment. Inflation at end-2009 should decline to 9 percent owing to the drop in commodity prices, and gross official reserves should decline to about 5.5 months of imports.

\section{A. Fiscal Policy}

13. Despite the international financial crisis and its impact on government finances, the government will implement all of the fiscal and public spending policies defined in the MEFP of January 8, 2009. The key objective of the 2009 spending policy remains to significantly improve the composition of public spending in favor of priority sectors in order to accelerate progress toward the Millennium Development Goals.

14. The international financial crisis will certainly impact government finances. It could result in a reduction in government revenues of about $1 \frac{1}{2}$ percentage points of GDP. To offset the impact of this crisis on the poor, emergency spending on targeted social safety nets (approximately 1.5 percent of GDP) could be implemented if additional budgetary assistance is obtained. Such spending will be used to finance food security and school feeding programs and for targeted assistance for the most vulnerable segments of the population and farmers.

15. The ratio of the government wage bill to GDP will decline less than anticipated in the medium term owing to higher payroll in the priority sectors, which are key for achieving progress toward the Millennium Development Goals. The ratio should remain below 11 percent of GDP toward the end of the program in 2011.

16. To contain the wage bill, the government will continue to draw on the findings of the Public Expenditure Management and Financial Accountability Review (PEMFAR) prepared jointly by the World Bank and the government of Burundi. In particular, with the census of civil servants having been completed and payroll management transferred to the Ministry of 
Finance, the government will proceed with the audit of the payroll to ensure that the calculated wages are indeed on a sound legal basis and that there are no abuses. This audit should precede the exercise to harmonize wages. To increase spending in the priority sectors, the government will implement a rationalization plan for nonpriority spending, in accordance with the PFM strategy.

17. In support of its fiscal policy, the government will strengthen the sliding quarterly cash flow plan which was partially implemented (end-June 2009 structural benchmark). Expenditure commitment and cash flow plans will be harmonized at the beginning of each quarter and submitted to the Minister of Finance for approval. In this context, all budget spending will require the prior authorization of the Minister of Finance and will be executed strictly on the basis of revenue availability. A monthly budget allocation for each ministry should be defined, with strict expenditure prioritization. To protect priority spending, the government will identify in advance nonpriority expenditure that will be cut in the event of a decline in revenue or financing.

\section{B. Monetary and Foreign Exchange Policy}

18. The government plans to pursue a prudent monetary policy, which is necessary to limit inflationary expectations. Given the improved inflation outlook, the BRB will ease its monetary policy. However, until the objective of single-digit inflation is attained, the central bank will set broad money growth below nominal GDP growth.

19. Better fiscal and monetary policy coordination will be essential, especially to coordinate responses to the impact of the international financial crisis. The central bank and Ministry of Finance will therefore hold monthly meetings. In addition, the role of the Cash Flow Management Committee will be strengthened with the preparation of monetary and fiscal policy recommendations to the BRB and the Ministry of Finance.

20. Proactive management of the central bank's foreign exchange reserves and sterilization of foreign exchange operations will continue within a floating exchange rate regime for the Burundi franc, with a view to achieving the inflation target. With technical assistance from the IMF, the central bank will continue to implement the recommendations of IMF experts for improving the operation of the foreign exchange market. In particular, in consultation with the commercial banks, the central bank will prepare and adopt a market convention with a code of conduct for market dealers. It will also conduct a critical annual general review of all aspects of foreign exchange reserve management.

\section{Structural Reforms}

21. In close collaboration with development partners, the government will continue to pursue its ongoing structural reforms: promotion of transparency and good governance, financial sector reform, regional integration, coffee sector reform, and oil sector reform. 
22. The reform program for 2009 as presented in the January 8, 2009, MEFP will be continued. In the fiscal area, the government will continue its gradual implementation of the new organic budget law and the PFM strategy and action plan, with the assistance of development partners, notably the IMF, the World Bank, and the European Union. The Ministry of Finance has already issued ministerial orders for creation of the entities that will implement the action plan. Moreover, the rationalization of ministerial accounts will continue as part of the gradual move toward a single Treasury account.

23. On the financial sector, although there are no signs of systemic risk resulting from the international financial crisis, the central bank will step up banking supervision on a preventive basis. In particular, the central bank will move ahead with the planned increase in the minimum capital requirements for banks to $\mathrm{FBu} 5$ billion and will take appropriate measures against banks that do not meet this requirement. Moreover, the central bank is awaiting the final recommendations of the study on the financial sector to guide its strategy and plan of action for reform of the sector.

24. With regard to regional integration, particularly with respect to the East African Community, a national strategy and action plan prepared with technical assistance from development partners will be adopted shortly.

25. With regard to the coffee sector, the process for the sale of coffee washing stations will move ahead following the creation of a regulatory authority for the coffee sector and publication of the related invitations to bid.

26. As for reform of the oil sector, the government will continue implementation of a price adjustment mechanism, in cooperation with the World Bank.

\section{External Financing}

27. The government will ensure that all its external obligations are settled when due. The Minister of Finance prepares monthly public debt position reports that give a detailed survey of obligations falling due. Regular publication of these reports is critical in strengthening Burundi's debt management.

28. Burundi will seek only concessional external financing or grants. The government will not contract nonconcessional foreign debt and will ensure that all loans contracted have a grant element of at least 50 percent. To make certain that the concessionality threshold is respected, the government will ensure compliance with the provision that the Ministry of Finance has the exclusive right to negotiate and sign external loans. 


\section{E. Technical Assistance and Coordination of Development Partners}

29. Burundi has vast technical assistance needs. The authorities plan to remain in close collaboration with bilateral and multilateral partners to build up the administrative capacity of the country's institutions. Technical assistance from development partners remains key in the areas of tax policy and administration, public expenditure management, monetary and foreign exchange policy, banking supervision, and economic statistics.

30. It is essential to coordinate relations with development partners, considering that they finance a major portion of budget expenditure. The government has stepped up its efforts to set up an institutional framework for coordination of assistance, namely the National Assistance Coordination Committee (CNCA). This initiative is supported financially by development partners. The CNCA can help:

- $\quad$ Organize the work between the government and development partners at the sectoral level, relying on the lead donor among the development partners for each sector. A high priority should be the creation of sectoral groups, as described in the CNCA organization chart.

- $\quad$ Centralize coordination of assistance within a single agency, which would facilitate coordination and decision-making by the government.

- $\quad$ Monitor aid disbursement and project implementation, in close collaboration with the Minister of Finance, to ensure that all financial assistance from development partners is included in the budget.

\section{F. Program Monitoring}

31. Program implementation will continue to be monitored on the basis of half-yearly reviews of the performance criteria, indicative targets, and structural benchmarks, as shown in Tables I.2 and I.3. The information to be reported to the IMF and the definition of the pertinent variables can be found in the attached Technical Memorandum of Understanding. Program implementation, achievement of the related objectives, and compliance with the performance criteria will be the subject of half-yearly reviews. The authorities also stand ready to adopt, in consultation with IMF staff, any further financial or structural measures that may prove necessary for the success of the program. 
Table I.1. Burundi: Performance Criteria and Indicative Targets for 2008

(Fbu billion, unless otherwise indicated)

\begin{tabular}{|c|c|c|c|c|c|c|c|c|c|c|c|}
\hline & \multirow{3}{*}{ 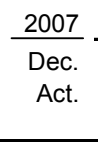 } & \multicolumn{10}{|c|}{2008} \\
\hline & & \multirow{2}{*}{$\frac{\text { Mar. }}{\text { Act. }}$} & \multicolumn{3}{|c|}{ Jun. $^{1}$} & \multicolumn{3}{|c|}{ Sep. } & \multicolumn{3}{|c|}{ Dec. $^{1}$} \\
\hline & & & Prog. & $\begin{aligned} \text { Prog. } \\
\text { Adj. }\end{aligned}$ & Act. & Prog. & $\begin{array}{r}\text { Prog. } \\
\text { Adj. }\end{array}$ & Act. & Prog. & $\begin{array}{r}\text { Prog. } \\
\text { Adj. }\end{array}$ & Act. \\
\hline \multicolumn{12}{|l|}{ Performance Criteria } \\
\hline Net foreign assets of the BRB (floor; US $\$$ million) ${ }^{2}$ & 67.5 & 59.3 & 67.4 & 55.7 & 53.1 & 15.9 & -3.4 & 63.1 & 16.9 & 16.9 & 112.9 \\
\hline Net domestic assets of the BRB (ceiling) ${ }^{2}$ & 49.4 & 48.4 & 51.6 & 65.2 & 71.6 & 124.9 & 146.9 & 71.3 & 117.1 & 117.1 & 17.0 \\
\hline Net domestic financing of the government (ceiling) ${ }^{2}$ & 8.5 & 19.1 & 37.3 & 50.9 & 37.7 & 21.6 & 43.6 & 26.0 & 13.2 & 13.2 & 19.0 \\
\hline External payments arrears of the government (ceiling; US $\$$ million) ${ }^{3}$ & 0.0 & 0.0 & 0.0 & & 0.0 & 0.0 & & 0.0 & 0.0 & & 0.0 \\
\hline Short-term external debt of the government (ceiling; US $\$$ million) ${ }^{3}$ & 0.0 & 0.0 & 0.0 & & 0.0 & 0.0 & & 0.0 & 0.0 & & 0.0 \\
\hline \multicolumn{12}{|l|}{$\begin{array}{l}\text { Nonconcessional external debt contracted or guaranteed by the } \\
\text { government or the BRB (ceiling; cumulative }\end{array}$} \\
\hline from the beginning of the calendar year; US $\$$ million $)^{3}$ & 0.0 & 0.0 & 0.0 & & 0.0 & 0.0 & & 0.0 & 0.0 & & 0.0 \\
\hline \multicolumn{12}{|l|}{ Indicative targets } \\
\hline Government's wage bill (ceiling; cumulative from beginning of calendar year) & 114.0 & 34.2 & 70.3 & & 70.8 & 106.3 & & 108.7 & 141.2 & & 154.7 \\
\hline Accumulation of domestic arrears (ceiling; cumulative from beginning of calendar year) & 0.0 & 0.5 & 0.0 & & 2.8 & 0.0 & & 3.3 & 0.0 & & 2.3 \\
\hline
\end{tabular}

1 Indicative targets.

${ }^{2}$ The ceiling or the floor will be adjusted as indicated in the TMU.

${ }^{3}$ Continuous performance criterion. 
Table I.2. Burundi: Performance Criteria and Indicative Targets for 2009 (Fbu billion, unless otherwise indicated)

\begin{tabular}{|c|c|c|c|c|c|c|c|c|c|c|}
\hline & \multirow{3}{*}{$\begin{array}{c}2008 \\
\text { Dec. } \\
\text { Act. }\end{array}$} & \multicolumn{9}{|c|}{2009} \\
\hline & & \multicolumn{3}{|c|}{ Mar. } & \multicolumn{2}{|c|}{ Jun. $^{1}$} & \multicolumn{2}{|c|}{ Sep. } & \multicolumn{2}{|c|}{ Dec. $^{1}$} \\
\hline & & Prog. & $\begin{array}{r}\text { Prog. } \\
\text { Adj. }\end{array}$ & Act. & Prog. & $\begin{array}{l}\text { Rev. } \\
\text { Prog. }\end{array}$ & Prog. & $\begin{array}{l}\text { Rev. } \\
\text { Prog. }\end{array}$ & Prog. & $\begin{array}{r}\text { Rev. } \\
\text { Prog. }\end{array}$ \\
\hline \multicolumn{11}{|l|}{ Performance Criterion } \\
\hline Net foreign assets of the BRB (floor; US\$ million) ${ }^{2}$ & 112.9 & 15.4 & -34.4 & 87.9 & 10.4 & 35.0 & 11.0 & 40.0 & 21.6 & 69.0 \\
\hline Net domestic assets of the BRB (ceiling) ${ }^{2}$ & 17.0 & 126.7 & 185.9 & 33.6 & 146.8 & 122.6 & 155.4 & 127.4 & 147.2 & 90.2 \\
\hline Net domestic financing of the government (ceiling) ${ }^{2}$ & 19.0 & 36.5 & 95.7 & -4.7 & 46.9 & 46.9 & 61.2 & 61.2 & 0.0 & 21.7 \\
\hline External payments arrears of the government (ceiling; US $\$$ million) ${ }^{3}$ & 0.0 & 0.0 & & 0.0 & 0.0 & 0.0 & 0.0 & 0.0 & 0.0 & 0.0 \\
\hline $\begin{array}{l}\text { Short-term external debt of the government (ceiling; US } \$ \text { million) }{ }^{3} \\
\text { Nonconcessional external debt contracted or guaranteed by the } \\
\text { government or the BRB (ceiling; cumulative }\end{array}$ & 0.0 & 0.0 & & 0.0 & 0.0 & 0.0 & 0.0 & 0.0 & 0.0 & 0.0 \\
\hline from the beginning of the calendar year; US $\$$ million $)^{3}$ & 0.0 & 0.0 & & 0.0 & 0.0 & 0.0 & 0.0 & 0.0 & 0.0 & 0.0 \\
\hline \multicolumn{11}{|l|}{ Indicative targets } \\
\hline Government's wage bill (ceiling; cumulative from beginning of calendar year) & 154.7 & 38.9 & & 31.4 & 86.7 & 91.8 & 141.7 & 155.8 & 180.4 & 192.2 \\
\hline Accumulation of domestic arrears (ceiling; cumulative from beginning of calendar year) & 2.3 & 0.0 & & 0.1 & 0.0 & 0.0 & 0.0 & 0.0 & 0.0 & 0.0 \\
\hline Reserve money (ceiling) & $\ldots$ & 145.0 & & 138.7 & 159.2 & 164.7 & 168.4 & 175.8 & 172.3 & 177.5 \\
\hline
\end{tabular}

1 Indicative targets.

${ }^{2}$ The ceiling or the floor will be adjusted as indicated in the TMU.

${ }^{3}$ Continuous performance criterion. 
Table I.3. Burundi: Performance Criteria and Structural Benchmarks for 2009

\begin{tabular}{lll}
\hline Measures & $\begin{array}{l}\text { Condition type Status } \\
\text { and date }\end{array}$ & Macroeconomic Rationale \\
\hline
\end{tabular}

\section{Fiscal management}

Resumption of payroll management by the Ministry of Finance by taking charge of the payroll database.

Close and transform into subaccounts for special allocation from the general treasury account the off-budget accounts mentioned in the finance minister's letter No. $540 / 4904 / 2008$ of November 12, 2008.

Close the accounts mentioned in the finance minister's letter No. $540 / 4768 / 2008$ of October 31, 2008.

Prepare and implement a sliding quarterly cash-flow plan.

\section{Revenue administration and tax policy}

Introduce VAT and the common external tariff.

\section{Fiscal governance}

In accordance with the laws of Burundi, the Fbu 6 billion and the deeds for 25 properties belonging to INTERPETROL that have been placed under seal will remain in place until a court decision has been reached on the INTERPETROL case.
Performance criterion Completed

(March 31, 2009)

Structural benchmark

(September 30, 2009)

Structural benchmark

(September 30, 2009)

Structural benchmark

(June 30, 2009)

Structural benchmark (July 31, 2009)

Structural benchmark Completed (continuous)
Efficient wage bill management is essential for focusing spending in priority areas, while enhancing fiscal sustainability. Wage bill management is being reinforced through the creation of a single wage data management and elimination of ghost employees.

Implementation of a single treasury account is key to sound public financial management.

Implementation of a single treasury account is key to sound public financial management.

Partially completed.

Enhance budget execution and ensure coordination of monetary and budget policies.
To raise the efficiency of tax collection, and offset potential losses on customs revenue due to the accession to EAC.

Enhance fiscal governance to ensure continuation of budget support. 


\section{APPENDIX I \\ ATTACHMENT II \\ TRANSLATED FROM FRENCH}

\section{BURUNDI}

TECHNiCAL MEMORANDUM OF UNDERSTANDING

1. This technical memorandum of understanding covers the agreements on monitoring implementation of the program supported by the Poverty Reduction and Growth Facility (PRGF) arrangement. It sets out the definitions of program variables to monitor implementation of the program and the reporting requirements for the government of Burundi and the Bank of the Republic of Burundi (BRB). It defines quantitative performance criteria, indicative targets, and applicable adjusters.

\section{A. Quantitative Program Targets}

\section{Quantitative performance criteria and indicative targets}

2. The quantitative performance criteria for the program as shown in the MEFP are as follows:

- $\quad$ net foreign assets of the BRB (floor);

- $\quad$ net domestic assets of the BRB (ceiling);

- $\quad$ net domestic financing of the government (ceiling);

- $\quad$ external payment arrears of the government (ceiling; continuous);

- $\quad$ stock of short-term external debt (maturity of less than one year) of the government and the BRB (ceiling; continuous); and

- new nonconcessional medium- and long-term external debt contracted or guaranteed by the government or the BRB (ceiling; continuous).

3. The quantitative indicative targets for the program, shown in the MEFP, are as follows:

- $\quad$ the government's wage bill (ceiling);

- $\quad$ accumulation of domestic arrears (ceiling); and

- $\quad$ reserve money (ceiling). 


\section{Definitions and measurement}

4. The net foreign assets of the BRB are defined as the difference between (i) gross official reserves (valued at market prices) and other claims; and (ii) foreign exchange liabilities to nonresident entities (including the use of Fund resources, and liabilities arising from the use of any SDR allocation(s)). The gross official reserves of the BRB are defined as those foreign assets that are liquid and freely available to the central bank.

5. The net domestic assets of the BRB are defined as the difference between (i) reserve money, comprising currency in circulation, reserves of commercial banks, and other deposits held at the BRB; and (ii) net foreign assets of the BRB.

\section{Adjuster for changes in the compulsory reserves coefficients}

6. The ceiling on net domestic assets of the BRB will be adjusted symmetrically for any change in the compulsory reserves coefficient applied to deposits in commercial banks, by the amount of the new coefficient minus that stipulated in the program, multiplied by bank deposits subject to compulsory reserves. The rate stipulated in the program is currently 3 percent.

7. Net domestic financing of the government is defined as the change in (i) outstanding loans, advances, and other credit to the government from the BRB and all of Burundi's commercial banks; (ii) the stock of all government securities held by the nonbank public denominated in Burundi francs, including that held by nonresidents; (iii) less government deposits held in the BRB or in Burundi's commercial banks. The coverage of government is defined as central government and any other special funds or operations that are part of the budgetary process or have a direct impact on the government's financial position.

8. The stock of external payments arrears for program monitoring purposes is defined as the end-of-period amount of external debt service due and not paid, including contractual and late interest, for which a clearance agreement is not in place or for which arrears are not reschedulable. Arrears for which a clearance framework has been agreed with the creditor or which are subject to rescheduling or restructuring are not considered arrears for program monitoring purposes. Program arrears would include any debt service due under such agreements that have not been paid.

9. The program includes a ceiling on new nonconcessional external debts contracted or guaranteed by the government and the BRB. This performance criterion applies to the contracting or guaranteeing by the central government, local governments, or the BRB of new nonconcessional external debt (as specified below) with an original maturity of one year or more, including commitments contracted or guaranteed for which value has not been received. The term "debt" shall be understood as defined in the Executive Board Decision 
No. 12274-(00/85) adopted August 24, 2000. Debt rescheduling and restructuring are excluded from the criterion. Included are financial leases and other instruments giving rise to external liabilities, contingent or otherwise, on nonconcessional terms. In determining the level of concessionality of these obligations, the definition of concessional borrowing shall apply. Concessional debt is defined as having a grant element of 50 percent or more. Management fees would also be taken into account when determining a loan's grant element. For loans with a maturity of at least 15 years, the 10 -year average commercial interest reference rates (CIRRs) published by the OECD should be used as the discount rate for assessing the level of concessionality, while the 6-month average CIRRs should be used for loans with shorter maturities. To both the 10-year and the 6-month average CIRRs, the following margins should be added: 0.75 percent for repayment periods of less than 15 years; 1 percent for 15-19 years; 1.15 percent for 20-29 years; and 1.25 percent for 30 years or more. The performance criterion is defined to exclude the use of Fund resources and any Burundi franc-denominated treasury securities held by nonresidents.

10. The stock of short-term external debt with a maturity of less than one year, owed by the central government is to remain at zero under the program. Normal import credits are excluded from this ceiling. Loans with an initial maturity, as recorded in the original loan agreement, of one year or more are considered medium-term or long-term loans. This performance criterion applies not only to debt, as defined in point No. 9 of the Guidelines on Performance Criteria with Respect to Foreign Debt, adopted August 24, 2000, but also to commitments contracted or guaranteed for which value has not been received (including leasing). Excluded from this performance criterion are rescheduling arrangements, borrowing from the Fund and any Burundi franc-denominated treasury securities held by nonresidents. As of September 2007, the stock of short-term debt outstanding was nil.

11. The government's wage bill is defined as total labor remunerations on a commitments basis for civil servants, contractual employees, police, and military personnel of the government, including all allowances and bonuses.

12. The accumulation of domestic arrears is measured by the accumulation of nonexecuted payment orders older than 60 days.

\section{External financial assistance adjustor}

13. The program provides for adjusters to allow higher than expected external assistance to be spent (with a cap) and shortfall of external assistance to be financed domestically (with a cap).

14. Any financing excess up to US $\$ 60$ million will be spent on expenditure priorities defined in the PRSP. The floor on the stock of net foreign assets of the central bank will be adjusted upward, and the ceilings on the net domestic assets of the central bank and on the 
net domestic financing to the government will be adjusted downward to accommodate 100 percent of any financing excess above US\$60 million.

15. The floor on the stock of net foreign assets of the central bank will be adjusted downward, and the ceilings on the net domestic assets of the central bank and on the net domestic financing to the government will be adjusted upward to accommodate a financing shortfall up to a maximum of US\$60 million. External financial assistance will be converted to Burundi francs using the program-specified $\mathrm{FBu} / \mathrm{US} \$$ exchange rate.

16. External financial assistance (measured in US\$) is defined to include the following: (i) nonproject loans and grants to the budget (including payments made through the multidonor trust fund managed by the World Bank for current debt service to multilaterals); (ii) debt relief on current maturities; minus (iii) any cash payments for external arrears clearance operations. Donor disbursements into blocked accounts for the purpose of clearing arrears will not be included as foreign assistance for program monitoring purposes.

\section{B. Provision of Information to IMF Staff}

17. To facilitate the monitoring of program implementation, the authorities will prepare and forward to the IMF African Department a monthly progress report on the program, within six weeks of the end of each month, containing

18. The following weekly data:

- $\quad$ foreign exchange auction market (MED) transactions;

- $\quad$ the balance sheet of the BRB (weekly statement) (BRB Research Department).

19. The following monthly data, with a maximum lag of six weeks:

- a monitoring table (tableau de bord) containing the most recent weekly and monthly data on the main financial indicators (REFES);

- $\quad$ a table on the foreign exchange cash flow (BRB Foreign Banking Operations Department);

- $\quad$ the monetary survey, including the breakdown of the central bank and of commercial banks (BRB Research Department);

- $\quad$ monthly exchange-rate data (official and parallel markets, end-of-month and monthly average) (BRB Research Department);

- $\quad$ a detailed breakdown of government revenue (Ministry of Finance); 
- a detailed breakdown of government expenditure on a commitment basis, including propoor spending (Ministry of Finance);

- a detailed breakdown of the servicing of domestic and external public debt, including amounts due and paid, in interest and principal, as well as the breakdown by creditor and any accumulation of arrears on domestic or external debt (Ministry of Finance);

- $\quad$ a detailed breakdown of the stock of domestic payments arrears for the current fiscal year (Ministry of Finance);

- the amount of new debts contracted or guaranteed by the government, including detailed information on the terms (such as currency denomination, interest rate, grace period, maturity) (Ministry of Finance);

- $\quad$ actual disbursements of nonproject financial assistance, including new loans and debt relief granted by Burundi's external creditors (Ministry of Finance); and

- $\quad$ an update on the implementation of structural measures planned under the program (REFES).

20. The following quarterly data, with a maximum lag of six weeks:

- $\quad$ progress reports on the BRB's internal reforms, including each unit's action plans for the coming month (Reform Monitoring Committee, BRB).

21. SP/REFES/Ministry of Finance and BRB will also provide the African Department of the IMF with any information that is deemed necessary to ensure effective monitoring of the program. 


\title{
INTERNATIONAL MONETARY FUND
}

BURUNDI

\begin{abstract}
Second Review Under the Three-Year Arrangment Under the Poverty Reduction and Growth Facility and Request for Modification of Performance Criteria
\end{abstract}

\section{Informational Annex}

Prepared by the African Department

(In consultation with other departments)

June 29, 2009

- Relations with the Fund. Describes financial and technical assistance from the Fund and provides information on the safeguards assessment and exchange rate system. Outstanding purchases and loans amounted to SDR 51.39 million (66.7 percent of quota) at end-May 2009.

- Relations with the World Bank. Describes the World Bank Group's program and portfolio.

- Relations with the African Development Bank. Describes the AfDB Group's operations and portfolio.

- Statistical Issues. Assesses the quality of statistical data. Shortcomings in economic statistics are hampering surveillance. 


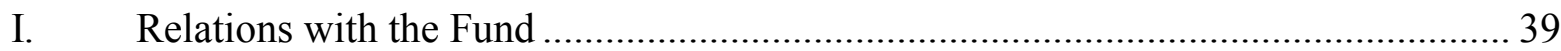

II. Joint World Bank-IMF Work Program, 2008-09 ............................................... 44

III. Relations with the African Development Bank Group ...................................... 45

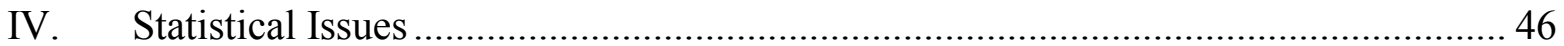




\section{APPENDix I \\ BURUNDI \\ RELATIONS WITH THE FUND}

(As of May 31, 2009)

I. Membership Status: Joined: September 28, 1963

$\underline{\text { Article XIV }}$

II. General Resources Account:

SDR Million

77.00

\% Quota

Quota

76.64

100.00

Fund holdings of currency

0.36

99.53

Reserve position

Holdings exchange rate

III. SDR Department:

SDR Million

\% Allocation

Net cumulative allocation

13.70

100.00

Holdings

0.04

0.31

IV. Outstanding Purchases and Loans:

PRGF arrangements

SDR Million

\% Quota

51.39

66.74

V. Latest Financial Arrangements:

\begin{tabular}{lcccc} 
Type & $\begin{array}{c}\text { Date of } \\
\text { Arrangement }\end{array}$ & $\begin{array}{c}\text { Expiration } \\
\text { Date }\end{array}$ & $\begin{array}{c}\text { Amount Approved } \\
\text { (SDR Million) }\end{array}$ & $\begin{array}{c}\text { Amount Drawn } \\
\text { (SDR Million) }\end{array}$ \\
\cline { 2 - 3 } PRGF & Jul 07, 2008 & Jul 06, 2011 & 46.20 & 13.20 \\
PRGF & Jan 23, 2004 & Jan 22, 2008 & 69.30 & 69.30 \\
PRGF & Nov 13, 1991 & Nov 12, 1994 & 42.70 & 17.21
\end{tabular}

VI. Projected Payments to Fund ${ }^{1}$

(SDR Million; based on existing use of resources and present holdings of SDRs):

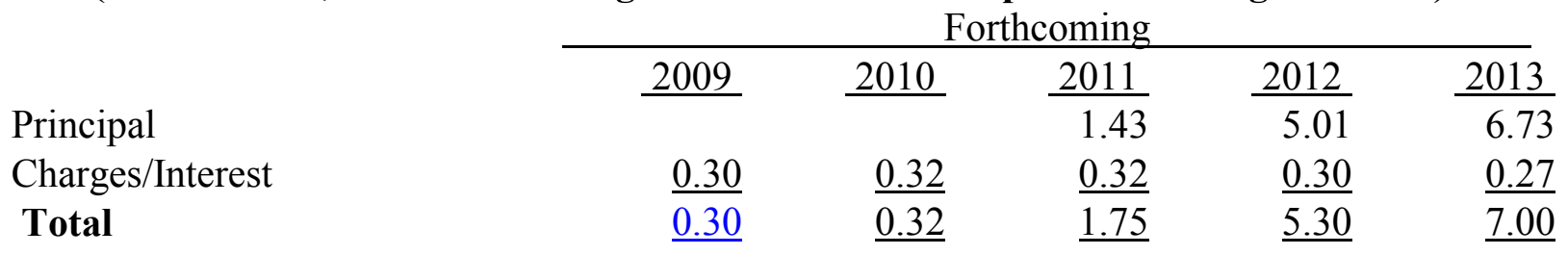

${ }^{1}$ When a member has financial obligations overdue for more than three months, the amount of such arrears will be shown in this section. 


\section{Safeguards Assessments}

An update assessment of the Banque de la République du Burundi (BRB) completed in June 2008 found that since the previous assessment, certain safeguards had been strengthened (e.g., external audits have been completed on a timely basis and audited financial statements comply with IFRS and are published). However, it also identified significant control weaknesses and recommended more robust controls over domestic disbursements to the government and its creditors, including contracting with an external auditor to review such controls (prior actions for the approval of the arrangement). Other safeguards recommendations include a system to monitor the status of audit and safeguards recommendations, continuation of semi-annual audits of disbursements to the government, and issuing guidelines for investment operations.

\section{Exchange Arrangements}

Burundi maintains a floating exchange regime. The U.S. dollar is the intervention currency. On June 25, 2009, the exchange rate was Fbu 1,220 to the dollar. In 2003 the central bank eliminated most remaining exchange restrictions on current international transactions and delegated authority to commercial banks to approve standard transactions. In early 2004, the surrender requirement was lowered to 50 percent and in early 2005 it was eliminated. The central bank has admitted foreign exchange bureaus to the weekly auctions. Most external arrears to bilateral and multilateral creditors were cleared by the end of 2005. In December 2006 the government published a new foreign exchange regulation that liberalized foreign exchange for current transactions and removed one multiple currency practice.

Burundi availed itself of the transitional arrangements of Article XIV when it joined the Fund in 1962 but no longer maintains any exchange restrictions or multiple currency practices that relate to that article. It does have one multiple currency practice that is inconsistent with Article VIII, Section 2(a): the exchange rate used for government transactions may differ by more than 2 percent from market exchange rates. Burundi maintains certain foreign exchange restrictions for security reasons and has notified the Fund of those restrictions pursuant to Decision 144-(52/51). The authorities have not requested, and staff does not propose, approval of the multiple currency practice. 


\section{Article IV Consultation}

In accordance with Decision 12794-(02/76), as amended by Decision 12854-(02/96), Burundi is on the 24-month Article IV cycle. The 2008 Article IV consultation was completed by the Executive Board on July 7, 2008 (IMF Country Report 08/282), along with the request of a new PRGF arrangement.

In concluding the 2008 consultation, Executive Directors acknowledged the commendable progress that Burundi made in implementing its first PRGF-supported program in a difficult postconflict environment. They agreed that fiscal sustainability in the face of a heavy debt burden will depend on broadening the revenue base and improving the composition of spending while financing the budget though grants and highly concessional external resources. Recognizing the recent acceleration of inflation deriving from rising international food and oil prices, Directors encouraged the monetary authorities to act to anchor inflation expectations and contain secondround effects of food and oil price shocks. Directors also saw the need to accelerate structural reforms, especially in the coffee sector, and welcomed Burundi's membership in the East African Community. 


\section{Technical Assistance}

\begin{tabular}{|c|c|}
\hline June 2009 & MCM multitopic mission \\
\hline April 2009 & FAD mission on implementing the organic budget law \\
\hline March 2009 & MCM mission on monetary operations \\
\hline March 2009 & MCM mission on foreign exchange \\
\hline March 2009 & MCM mission on internal audit \\
\hline March 2009 & MCM mission on reserve management \\
\hline February 2009 & AFRITAC mission on banking regulation and supervision \\
\hline February 2009 & FAD PSIA mission on fuel pricing policy and social protection \\
\hline January 2009 & MCM FSAP mission \\
\hline December 2008 & AFRITAC mission on fiscal administration \\
\hline December 2008 & MCM multitopic mission \\
\hline December 2008 & MCM mission on internal audit \\
\hline August 2008 & MCM mission on organizational, human resources, and communication reforms \\
\hline July 2008 & MCM mission on foreign exchange \\
\hline June 2008 & AFRITAC mission on banking regulation and supervision \\
\hline May 2008 & FAD mission for installation of a PFM resident advisor \\
\hline January 2008 & FIN safeguards assessment mission \\
\hline December 2007 & AFRITAC statistics mission on national accounts and consumer price index \\
\hline November/December 2007 & STA monetary and financial statistics mission \\
\hline November 2007 & AFRITAC mission on building capacity in banking supervision \\
\hline November 2007 & FAD Tax policy mission \\
\hline November 2007 & STA monetary statistics mission \\
\hline September 2007 & MCM multitopic mission \\
\hline September 2007 & AFRITAC mission on tax revenue administration \\
\hline September 2007 & AFRITAC mission on building the capacity of the central bank \\
\hline September 2007 & FAD public financial management mission \\
\hline July 2007 & AFRITAC PFM mission on payroll control \\
\hline June 2007 & AFRITAC PFM mission on treasury operation and control \\
\hline June 2007 & MCM technical assistance mission \\
\hline June 2007 & AFRITAC mission on building debt management capacity \\
\hline March 2007 & FAD resident expert on public accounting \\
\hline December 2006 & FAD public expenditure management mission \\
\hline November 2006 & MCM banking supervision mission \\
\hline September 2006 & FAD customs and tax administration mission \\
\hline April 2006 & MCM foreign exchange reserve management mission \\
\hline March 2006 & STA mission to prepare the metadata and medium-term action plan \\
\hline March 2006 & MCM/LEG joint Article VIII mission \\
\hline January 2006 & LEG AML/CFT legislative drafting mission \\
\hline January 2006 & Monetary operations/FOREX/banking supervision mission \\
\hline
\end{tabular}


XI. Implementation of HIPC Initiative:

Enhanced

I. Commitment of HIPC assistance

Framework

Decision point date

Aug 2005

Assistance committed

by all creditors (US\$ million) ${ }^{1}$

832.6

of which: IMF assistance (US\$ million)

27.87

(SDR equivalent in

millions)

19.28

Completion point date

Jan. 2009

II. Disbursement of IMF assistance (SDR million)

Assistance disbursed to the member

Interim assistance

Completion point balance

19.02

Additional disbursement of interest income ${ }^{2}$

Total disbursements

22.35

\footnotetext{
${ }^{1}$ Assistance committed under the original framework is expressed in net present value (NPV) terms at the completion point, and assistance committed under the enhanced framework is expressed in NPV terms at the decision point. Hence these two amounts cannot be added.

${ }^{2}$ Under the enhanced framework, an additional disbursement is made at the completion point corresponding to interest income earned on the amount committed at the decision point but not disbursed during the interim.
}

\section{Resident Representative:}

A part-time resident representative took up the post in May 2005 and an office with an administrative assistant opened in January 2006 in Bujumbura. Mr. Israel de la Piedra was the IMF's resident representative to Burundi from May 2007 to May 2009. 


\section{APPENDIX II}

\section{BURUNDI \\ JoINT WORLD BANK-IMF WORK PROGRAM, 2008-10}

\begin{tabular}{|c|c|c|c|}
\hline Title & Products & $\begin{array}{c}\text { Provisional Timing of } \\
\text { Mission }\end{array}$ & Expected Delivery Date \\
\hline & A. Mutual Information o & Relevant Work Programs & \\
\hline \multirow[t]{17}{*}{ Bank work program } & 1. Public Expenditure & July 2008 & August 2008 \\
\hline & Management and Financial & & \\
\hline & Accountability Review & & \\
\hline & (PEMFAR) & & \\
\hline & 2. Public Expenditure & Sept. 09 & April 2010 \\
\hline & Review & & \\
\hline & 3. ERSG-2 & March 17-28 (appraisal) & August 2008 \\
\hline & (Budget support) & & \\
\hline & 4. ERSG-3 & Sept. 08, Dec. 08. & August 2009 \\
\hline & (Budget support) & (identification) & \\
\hline & & March-April 09. & \\
\hline & & (pre- appraisal) & \\
\hline & & June 09 (appraisal) & \\
\hline & 5. ERSG-4 & Sept. 09-Nov. 09 & \\
\hline & (Budget support) & (Identification) & \\
\hline & 6. Country Economic & July 09 (main mission) & March-May 2010 \\
\hline & Memorandum (CEM) & & \\
\hline \multirow[t]{7}{*}{ IMF work program } & 1. Article IV consultation, & April 30-May 14 and & July 2008 \\
\hline & negotiations for new PRGF & May 25-31, 2008 & \\
\hline & arrangement & & \\
\hline & 2. First PRGF review & November 2008 & January 2009 \\
\hline & 3. Second PRGF review & May 2009 & July 2009 \\
\hline & 4. Third PRGF review & November 2009 & January 2010 \\
\hline & 5. Fourth PRGF review & May 2010 & July 2010 \\
\hline \multicolumn{4}{|c|}{ B. Requests for Work Program Inputs } \\
\hline Fund request to Bank & $\begin{array}{l}\text { 1. Policy note on domestic } \\
\text { petroleum sector }\end{array}$ & & $\begin{array}{l}\text { December } 2008 \text { (detailed } \\
\text { background report) and } \\
\text { May } 2009 \text { (synthesis report) }\end{array}$ \\
\hline \multirow[t]{4}{*}{ Bank request to Fund } & $\begin{array}{l}\text { 1. Study of exchange rate } \\
\text { misalignment and }\end{array}$ & & July 2008 \\
\hline & competitiveness & & \\
\hline & 2. Study on the sources of & & December 2009 \\
\hline & inflation in Burundi & & \\
\hline & C. Agreement on Joi & Products and Missions & \\
\hline \multirow[t]{4}{*}{ Joint products } & FSAP & & December 2008 \\
\hline & DSA & & December 2008 \\
\hline & JSAN on APR & & December 2008 \\
\hline & HIPC CP documents & & December 2008 \\
\hline
\end{tabular}




\section{APPENDIX III}

\section{BURUNDI \\ Relations With the African DeVelopment Bank Group}

(As of May, 26, 2009)

Burundi has been a member of the African Development Bank (AfDB) Group since the group was founded in 1964. AfDB grant and loan operations with the country were interrupted by the outbreak of civil strife in 1993. On July 19, 2004, the AfDB Boards approved general policy guidelines to help postconflict countries clear their arrears and created a facility, the PCCF, initially funded with about SDR 100 million in AfDB funds, to provide financial assistance to qualifying countries. The policy guidelines call for a three-way burden-sharing formula among the country, donors, and the PCCF. On October 27, 2004, the AfDB Boards endorsed an arrears clearance proposal for Burundi whereby the balance of arrears was settled with the help of donors and the PCCF before the decision point for the enhanced HIPC Initiative.

On $21^{\text {st }}$ April 2009, under the enhanced framework of the Heavily Indebted Poor Countries (HIPC) Initiative, the Board of Directors of AfDB approved AfDB's share of the HIPC debt relief in an amount equivalent to USD 150,200,000 in Net Present Value (NPV) terms as of the end of 2004. The Board of Directors also approved Burundi's qualification for debt relief under the Multilateral Debt Relief Initiative (MDRI), in an amount of US\$15.38 million, (UA 10.48 million), in nominal terms.

The following table provides an overview of AfDB's current operations and pipeline in Burundi.

\begin{tabular}{|c|c|c|c|}
\hline Sector & Name & Status & Amount \\
\hline & & & $\begin{array}{c}\text { UA } \\
\text { millions }\end{array}$ \\
\hline Rural development & Watershed management & ongoing & 9.00 \\
\hline Water and sanitation & Rehabilitation and extension project & ongoing & 12.00 \\
\hline Energy & Rehabilitation and extension project & ongoing & 7.30 \\
\hline Social & Multisectoral reinsertion project & ongoing & 9.80 \\
\hline Transport & Kirundo-Gasenyi road & ongoing & 14.90 \\
\hline Governance & Institutional capacity building project & ongoing & 2.10 \\
\hline Governance & Governance structures support program & ongoing & 1.50 \\
\hline Governance & Economic reforms support program (PARE II) & ongoing & 14.00 \\
\hline Governance & $\begin{array}{l}\text { Addition to the Economic reforms support program } \\
\text { (PARE II) }\end{array}$ & preparation & 10.00 \\
\hline Rural development & Multinational : Rural development in Bugesera & preparation & 15.00 \\
\hline Transport & Gitega-Ngozi road & preparation & 33.84 \\
\hline Transport & $\begin{array}{l}\text { Multinational : Mugina-Bujumbura-Ruhwa- } \\
\text { Ntendezi-Gisenyi road }\end{array}$ & ongoing & 49.50 \\
\hline Job creation & $\begin{array}{l}\text { Ngozi road pavement, education infrastructures } \\
\text { and watershed management }\end{array}$ & ongoing & 10.00 \\
\hline Energy & Multinational : power interconnection BI-NELSAP & ongoing & 15.00 \\
\hline & Total & & 203.94 \\
\hline
\end{tabular}




\section{APPENDIX IV}

\section{BURUNDI \\ STATISTICAL ISSUES}

\section{AsSessment of Data Adequacy for Surveillance}

\section{General}

Data provision has serious shortcomings that significantly hamper surveillance. The most acute shortcomings affect national accounts, government finance, and balance of payments statistics. STA missions have found that staffing shortages, insufficient funding, and lack of equipment impede production and dissemination of macroeconomic statistics. Coordination among institutions responsible for compiling statistics has been poor. Burundi could benefit from participation in the General Data Dissemination System as a framework to improve its macroeconomic statistics and coordination among the institutions that compile it.

\section{National Accounts}

Serious deficiencies in real sector data handicap analysis and economic management. National accounts are compiled infrequently. Source data on agriculture, the most important activity, is inadequate. The Statistical Office (ISTEEBU) responsible for producing economic statistics and preparing national accounts has weak capacity. Since 1998 Burundi has reported annual national accounts estimates to the Fund with about a three-month lag, which are derived from a macroeconomic projection model maintained by the Ministry of Planning and Reconstruction. Recently, ISTEEBU has developed, with AFRISTAT's help, a set of provisional national accounts for 2005, the new base year. This is a major effort because the last national accounts data were from 1998.

\section{Price statistics}

The CPI is compiled monthly. Its data are now collected in provinces as well as the capital, Bujumbura. Weights, which are based on a 1991 household expenditure survey, are in the process of being revised. There are no producer price indices.

\section{Labor market statistics}

The data on employment are out of date.

\section{Government finance statistics}

The government compiles budgetary central government data using source data from the Ministry of Economy, Finance, and Development (MEFD). Until recently, ministries seldom maintained 
computerized ledgers, making it impossible to establish balances and other accounting controls. Little accounting information was available on extrabudgetary units. There were also problems in recording arrears on external debt and current expenditure financed by foreign grants, which led to significant discrepancies between the balance of revenue and expenditure and financing estimates. With World Bank and FAD assistance, improved public financial management information systems that generate, among other things, standard quarterly budget execution reports implemented in 2005 are now largely operational. STA has provided training in government finance statistics (GFS) to compilers both at the Joint Africa Institute and INS. Despite these initiatives, the authorities still report the Summary government finance statistics transactions data for publication in International Finance Statistics, four months after the reference month. They are yet to improve coverage, statistical techniques, periodicity, and timeliness of GFS according to Government Finance Statistics Manual 2001 framework.

\section{Monetary statistics}

An STA technical assistance mission November 22-December 4, 2007 helped the Bank of the Republic of Burundi (BRB) improve its monetary and financial statistics. As a result, the monetary statistics it compiles largely follow the Monetary and Financial Statistics Manual methodology, which should make it easier to harmonize Burundi's monetary statistics to those of other members of the East African Community. The BRB has completed migration to the Standardized Report Forms for the submission of its monetary statistics to the Fund, and they were published in the June 2008 issue of the IFS Monetary and Financial Statistics Supplement. With the support of the 2007 mission, the BRB compiled an enlarged broad money aggregate (M3) which includes deposits in foreign currencies.

\section{Balance of payments}

Annual balance of payments and international investment position statistics are compiled according to the fifth edition of the Balance of Payments Manual (BPM5). Merchandise trade statistics are derived from customs data. Coverage adjustments are made to customs data to account for imports of electricity and undeclared imports (5\%) and for exports of coffee and tea. Source data for transportation are derived from an annual survey of transportation companies. Travel is derived from a survey of hotels, augmented with bank-reported data. Data for other services are collected mostly from monthly bank settlement reports. Income estimates are almost exclusively derived from those reports. For both services and income, the accuracy of the source data is not routinely assessed against other data sources. Data on current and capital transfers, as well as on financial account transactions, are incomplete; further BRB action is needed to improve their quality and reliability. Balance of payments and international investment position data are published in International Financial Statistics and in the Balance of Payments Yearbook through 2007.

\section{DAta STANDARds ANd Quality}

Not a General Data Dissemination System participant. No data ROSC is available. 


\section{Burundi: Table of Common Indicators Required for Surveillance}

(As of June 15, 2009)

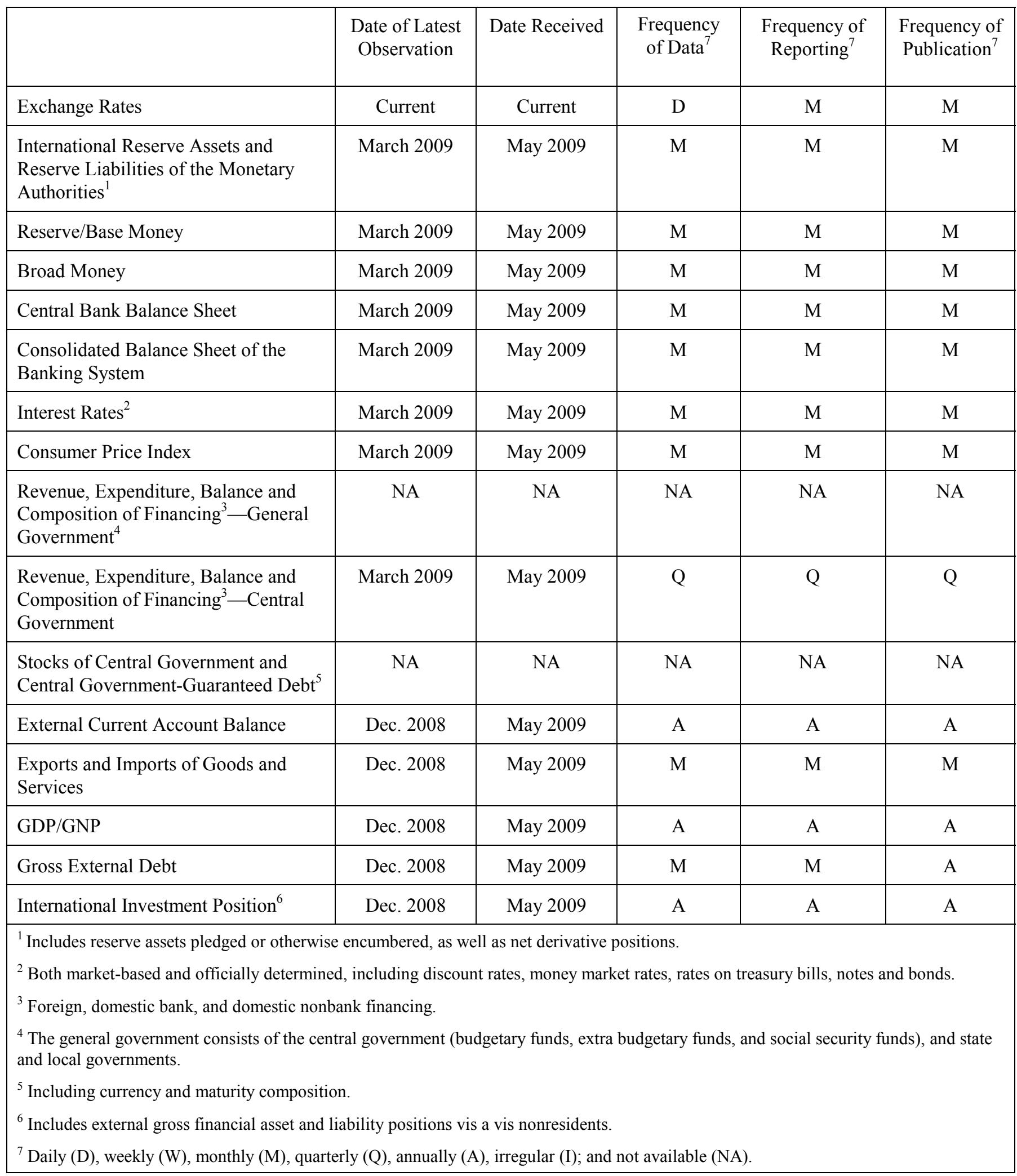


Press Release No.09/261

FOR IMMEDIATE RELEASE

International Monetary Fund

July 13, 2009

Washington, D.C. 20431 USA

\section{IMF Completes Second Review under PRGF Arrangement with Burundi and Approves US\$10.2 Million Disbursement}

The Executive Board of the International Monetary Fund (IMF) today completed the second review of Burundi's economic performance under the Poverty Reduction and Growth Facility (PRGF) arrangement. The completion of the review enables the immediate disbursement of an amount equivalent to SDR 6.6 million (about US\$10.2 million), bringing total disbursements to SDR 19.8 million (about US\$30.7 million).

The PRGF arrangement was approved on July 7, 2008 (see Press Release No 08/167) for an amount equivalent to SDR 46.2 million (about US\$71.6 million).

Following the Executive Board's discussion of Burundi's economic performance, Mr. Murilo Portugal, Deputy Managing Director and Acting Chair, said:

"The Burundian authorities are to be commended for their strong performance under the PRGF-supported program in a difficult post conflict environment. In particular, the valueadded tax was passed, payroll management transferred to the Ministry of Finance and the census of government employees completed. Monetary and financial sector reforms have also progressed well, and the foreign exchange auction system is being improved.

"The global economic crisis presents the Burundian authorities with macroeconomic challenges. To deal with these challenges, the revised program for 2009 provides for nearterm fiscal and monetary policy easing, anchored in a prudent medium-term strategy.

"Efforts to enhance revenue mobilization and to maintain the momentum of public financial management reforms, including the implementation of a single treasury account, will contribute to further strengthening macroeconomic stability. External financing of the budget should continue to be strictly limited to grants and highly concessional loans, given the high risk of debt distress. Monetary policy will aim to reduce inflation to low single digits.

"Efforts to strengthen the financial sector will aim at further improving banking supervision, developing a financial sector strategy, and enhancing central bank internal controls and risk management systems. The forthcoming Bank-Fund Financial Sector Assessment Program report will help guide these efforts. 
"The achievement of the country's medium-term objectives of sustained growth and poverty reduction will depend on accelerating structural reforms. Ongoing progress in reforming the coffee sector is welcome, and is key to reducing poverty. Burundi's East African Community membership will give further impetus to trade, structural reforms, and private sector development," added Mr. Portugal. 


\section{Statement by Samuel Itam, Executive Director for Burundi \\ July 13, 2009}

\section{INTRODUCTION}

My Burundian authorities have persevered with prudent macroeconomic policies and deepened structural reforms in spite of the challenging external environment and post conflict reconstruction effort. Reflecting this stance, they remain confident that the impacts of the external shocks on economic growth and balance of payments will be reversed in the medium-term as appropriate measures are implemented and the external environment improves. However, they concur with staff that the deteriorating external environment, coupled with the country's dependence on coffee exports for its foreign exchange revenue, poses considerable tail risks to the country's balance of payments outlook. In this regard, the authorities appreciate the constructive engagement and support of the Fund, the World Bank, the African Development Bank, and the donor community, and thank staff for their candid policy dialogue and advice under the program. The support and dialogue have been invaluable to the authorities' efforts to sustain the growth tempo and to respond to the challenges of the internal and external environment.

The authorities' perseverance in consolidating peace and macroeconomic stability strengthened the implementation of the PRGF-supported economic program. To this end, all end-March 2009 quantitative and structural performance criteria were met, except the target for cumulative domestic arrears that was missed because of delay in disbursement of budget support resources. Progress in the implementation of the reform agenda has been impressive, and the structural reforms are on track. In view of the strong performance under the program, the authorities request Directors' support for the completion of the second review of the PRGF arrangement, the third disbursement, modification of the performance criteria for end-September, and the conversion of all structural performance criteria into benchmarks.

\section{RECENT ECONOMIC DEVELOPMENTS}

Burundi's strong macroeconomic performance, which has been uninterrupted since 2006, anchored on deepened economic reforms, good coffee harvest and increased donor funded investment projects, is now threatened by the adverse effects of the global economic slowdown. Real GDP growth, estimated at 4.5 percent in 2008 on account of strong performance in the key economic sectors, is now projected to moderate to 3.2 percent in 2009 as a result of a severe external shock on Burundi's coffee exports and lower private transfers and foreign direct investment. On the external account the fall in coffee prices, transfers and FDI will be largely offset by the decline in the global prices of food and fuel. The overall balance of payments is forecast to register a small deficit, resulting in a slight decline in the official reserves, but remaining above 5 months of import cover. 
The deterioration in the fiscal outturn in 2008/09 reflects the challenges the country is facing. Revenue is estimated to stagnate relative to GDP, resulting in an increase in the fiscal deficit from 3 percent of GDP to 4.3 percent of GDP. This is despite the authorities' efforts to broaden the tax base, implement structural reforms, and strengthen customs and tax administration. To support the recovery of the economy, the authorities are determined to increase the level of spending under the major development and recurrent expenditure categories. However, they are aware and agree with staff that revenue shortfall may impact on the overall budget execution. To this end, they have agreed on a modest net domestic financing, coupled with easing of monetary policy.

The monetary framework continues to be guided by the objective of price stabilization, while allowing sufficient scope for economic growth. The central bank's monetary policy stance includes a mix of policy instruments for liquidity management. As a result, reserve money growth remained on track, and the banking system continued to meet the demand for private sector credit. Inflation, which had edged up in 2008 due to the surge in global food and fuel prices, is expected to moderate in response to declining global prices, and sustained prudent fiscal and monetary policies.

The country's financial system has remained resilient to the global financial crisis, mainly due to its limited exposure and well supervised and capitalized banks. This resilience was confirmed by the recent Bank-Fund FSAP mission that detected no obvious signs of imminent systemic stress. However, the banking system faces significant risks from the effects of the economic downturn. To this end, the authorities remain vigilant and ready to take necessary measures to protect the stability of the financial system.

\section{Policy ResPonse}

On the fiscal front, the authorities have opted for a small fiscal stimulus in order to minimize the adverse effects of the current economic slowdown, but remain committed to a near- and medium-term macroeconomic framework that aims at restoring growth to its previous trajectory. The authorities intend to consolidate current expenditure as a percentage of GDP, while increasing spending on priority sectors in line with the country's PRS. Spending would target improving access to basic social services, particularly in education and health, as well as improving access to public facilities like water, electricity and roads in the rural areas. The authorities will continue to pursue prudent fiscal policies in the medium term, aimed at limiting debt-creating domestic financing to the program target, while providing fiscal space for increased investments in agriculture and key MDG clusters.

To achieve these objectives, the authorities intend to slightly increase budgetary spending levels for the current financial year, in particular scaling-up spending on MDG clusters in line with their PRS, and supported by the HIPC and MDRI resources. To maintain spending at the budgeted levels and to boost spending on infrastructure and social programs, and others that directly raise agricultural productivity, the authorities will, in the near term, shift the fiscal anchor from zero net domestic financing to 0.7 percent of 
GDP. The authorities remain committed to taking additional fiscal measures to support the recovery of the economy and implement their poverty reducing policies. While aiming at providing adequate fiscal stimulus in light of the country's low level of public debt following the HIPC and MDRI debt relief, the authorities are mindful of the country's potential debt burden. In this regard, they remain committed to maintain debt sustainability by implementing fiscal reforms including a determined effort to further broaden the revenue base, enhanced mobilization of concessional resources and expenditure management.

To ensure budget credibility and make public spending more effective, the authorities have reaffirmed their commitment to enhance the management of public expenditure. They are dedicated to strengthening external debt management following the HIPC completion point and MDRI debt relief. To prevent recurrence of external debt stress, the authorities will pursue concessional borrowing and grants. The authorities concur with staff's recommendations for revamping the medium-term expenditure framework process to allow for strategic planning and ensuring that the budget allocations are consistent with the PRS objectives.

The authorities are confident, and staff concur, that domestic revenue mobilization effort should improve on the current revenue effort of 18 percent of GDP. This would entail broadening the tax base and strengthening fiscal controls, and reducing discretionary tax exemptions. The authorities will continue implementing the modernisation program for the tax revenue administration. The Parliament has passed the VAT law in line with the country's EAC partners, and the authorities have introduced the EAC common external tariff (CET). Other targets are to improve tax compliance, collect tax arrears, and phaseout the temporary suspension of tax levies on oil products.

These fiscal measures will be further supported by the easing of the stance of monetary policy. To achieve the objective of anchoring its monetary policy on low and stable inflation, the central bank will further bolster its open market operations, maintain a flexible exchange rate policy, rely substantially on foreign exchange sales for sterilization of liquidity, and continue to improve liquidity forecasting. The authorities reaffirm their commitment to target base money growth for containing inflation as an appropriate policy framework. Monetary policy stance will also help sustain the current level of credit to the private sector in the medium-term. The authorities have made a commitment to enhance coordination of their fiscal and monetary policies to enable them support economic recovery, at the same time achieving the objective of single digit inflation. To this end, coordination of fiscal and monetary policies will target at improving central bank liquidity forecasting and absorption of aid-financed expenditures.

The authorities have made impressive progress in implementing the structural reform agenda. Their agenda includes the reform of the financial sector, privatizing the coffee sector, the oil sector and promoting public accountability. To facilitate further reform of the public financial management, the Cabinet adopted a PFM strategy and action plan, and stepped-up rationalization of government bank accounts. The central bank will 
continue to strengthen its internal control and risk management system, and to align its open market operations to best practice. The central bank will also continue to spearhead the implementation of the financial sector reform strategy with Fund-Bank support. In this context, the authorities also appreciate the support from the development partners.

The authorities remain steadfast to maintaining a stable macroeconomic environment, creating conditions for sustained broad-based economic growth and reduction of poverty, and persevere with the structural reform agenda. Efforts to create employment opportunities through significant public investment in infrastructure and reforms to the business environment remain priorities. Trade liberalization under the auspices of the East African Community (EAC) is expected to assist in economic diversification and increased competition. Notwithstanding the strong policy framework and commitment to deepen structural reforms, and the positive medium term macroeconomic prospects, the authorities concur with staff assessment that the country faces a number of economic, political, social, security, and exogenous-related challenges going forward.

\section{CONCLUSION}

The economy of Burundi did not escape the impact of the global crisis. Coupled with the challenging internal environment, the risks to the economy and the program have been elevated. Nonetheless, my Burundian authorities are committed to the implementation of appropriate stabilization and structural policies. In the near-term, the authorities are determined to focus on sustaining macroeconomic stability, increasing domestic resource mobilization, promoting pro-poor growth, increasing investment in agriculture and key MDG clusters, and implement the power-sharing agreement to consolidate the peace process. Their commitment to the PRGF-supported program is unwavering and their endMarch 2009 program performance has been impressive. To this end, they request the completion of the second review under the PRGF arrangement and the third disbursement.

The authorities are confident that going forward, continued engagement with the Fund and the support of the development partners will enable them weather the adverse effects of the global crisis and achieve their development goals. It will also provide them with a sound basis for achieving some of the critical MDGs and consolidate the peace process. 